\title{
The Finite Volume-Complete Flux Scheme for Advection-Diffusion-Reaction Equations
}

\author{
J.H.M. ten Thije Boonkkamp • M.J.H. Anthonissen
}

Received: 1 October 2008 / Revised: 1 February 2010 / Accepted: 28 May 2010 /

Published online: 16 June 2010

(C) The Author(s) 2010. This article is published with open access at Springerlink.com

\begin{abstract}
We present a new finite volume scheme for the advection-diffusion-reaction equation. The scheme is second order accurate in the grid size, both for dominant diffusion and dominant advection, and has only a three-point coupling in each spatial direction. Our scheme is based on a new integral representation for the flux of the one-dimensional advection-diffusion-reaction equation, which is derived from the solution of a local boundary value problem for the entire equation, including the source term. The flux therefore consists of two parts, corresponding to the homogeneous and particular solution of the boundary value problem. Applying suitable quadrature rules to the integral representation gives the complete flux scheme. Extensions of the complete flux scheme to two-dimensional and time-dependent problems are derived, containing the cross flux term or the time derivative in the inhomogeneous flux, respectively. The resulting finite volume-complete flux scheme is validated for several test problems.
\end{abstract}

Keywords Advection-diffusion-reaction equation · Flux · Finite volume method · Integral representation of the flux $\cdot$ Numerical flux

\section{Introduction}

Conservation laws are ubiquitous in continuum physics, they occur in disciplines like fluid mechanics, combustion theory, plasma physics, semiconductor physics etc. These conservation laws are often of advection-diffusion-reaction type, describing the interplay between different processes such as advection or drift, diffusion or conduction and (chemical) reaction or recombination/generation. Examples are the conservation equations for reacting flow [21] or the drift-diffusion equations for semiconductor devices [11, 14].

Their numerical solution requires at least adequate space discretisation and time integration methods. For space discretisation there are many (classes of) methods available, such

J.H.M. ten Thije Boonkkamp ( $\varangle)$ · M.J.H. Anthonissen

Department of Mathematics and Computer Science, Eindhoven University of Technology,

P.O. Box 513, 5600 MB Eindhoven, The Netherlands

e-mail: tenthije@win.tue.nl 
as finite element, finite difference, finite volume or spectral methods. We restrict ourselves to finite volume methods (FVM); for a detailed account see e.g. [7, 17, 34]. Finite volume methods are based on the integral formulation, i.e., the conservation law is integrated over a disjunct set of control volumes covering the domain. The resulting (semi)discrete conservation law involves fluxes at the interfaces of the control volumes, which need to be approximated. For time integration there exist many sophisticated methods, for a detailed account see, e.g., [9].

Our objective in this paper is to present new expressions for the flux, which will subsequently be used to derive numerical flux approximations. We require that for onedimensional steady equations the numerical flux has the following properties. First, it should be unconditionally second order accurate, in particular, the flux approximation should remain second order accurate for highly dominant advection. This excludes the hybrid scheme of Spalding [27], which reduces to the standard upwind scheme when diffusion is absent. Second, the numerical flux should not produce spurious oscillations for dominant advection, as the standard central difference scheme does, and third, the flux may only depend on neighbouring values of the unknown, resulting in a three-point scheme. The latter requirement rules out high resolution schemes based on flux/slope limiters [13, 34] or (W)ENO reconstruction [25].

Our scheme is inspired by two papers by Thiart [30, 31]. In these papers a finite volume method is combined with an exponential scheme for the flux. More specifically, the fluxes at the cell interfaces are computed from a local boundary value problem, assuming piecewise constant coefficients. The source term is included in the computation of the fluxes. Similar schemes have been published in the last few decades. Without trying to be complete, we just mention a few. Allen and Southwell [1] and Il'in [10] introduced an exponentially fitted scheme, which is a hybrid central difference-upwind scheme such that the difference scheme locally has the same (exponential) solutions as the corresponding differential equation; see also [5] for a detailed account. An improvement of this scheme is proposed by El-Mistikawy and Werle [6]. These exponentially fitted schemes are a special case of the so-called locally exact schemes. The basic idea is to represent the solution in two adjacent intervals in terms of an approximate Green's function; see [17] and references therein. Exponentially fitted schemes are nowadays widely used to simulate advection-diffusion-reaction problems from continuum physics, especially to compute numerical solutions of the drift-diffusion model for semiconductor devices. For this application these schemes are known as the ScharfetterGummel scheme; see e.g. [3, 4, 24]. An extension of this scheme is due to Miller [16], who included the recombination term in the fluxes. A further extension to systems is presented in [33], where the avalanche generation source term is included in the numerical flux vector.

Our scheme is an extension of the schemes by Thiart. We derive an integral representation for the flux from the solution of a local boundary value problem (BVP) for the entire equation, including the source term, but we do not restrict ourselves to (locally) constant coefficients. As a consequence, the flux has a homogeneous and an inhomogeneous component, corresponding to the homogeneous and the particular solution of the boundary value problem, respectively. Suitable quadrature rules are applied to derive the numerical flux. The inclusion of the inhomogeneous flux will be of importance when advection dominates diffusion.

Extension of our scheme to two-dimensional equations is not just the separate application in $x$ - and $y$-direction. Instead, in order to accurately resolve the two-dimensional structure of the solution, we include the cross flux in the flux approximation. This means that for the computation of the $x$-component of the numerical flux, say, we put all $y$-derivatives in the right hand side and solve the resulting quasi-one-dimensional BVP. Therefore, the $x$ component of the flux will contain a part of the $y$-component. Mutatis mutandis, we derive 
the $y$-component of the flux. The resulting scheme is an upwind weighted space discretisation.

Likewise, for time-dependent problems, we include the time derivative in the source term and solve the resulting quasi-stationary BVP to derive the numerical flux. Consequently, the numerical flux contains the time derivative, resulting in an implicit ODE system. This semidiscretisation has usually much smaller dissipation and dispersion errors than the standard semidiscretisation, at least for smooth solutions. For high wave number solutions, as they might occur in discontinuities, say, also our scheme is prone to significant dispersion errors. For time integration of the semidiscretisation we can use any suitable method. In this paper we choose the trapezoidal rule.

Our scheme is suitable to discretise a large class of advection-diffusion-reaction equations. Especially for dominant advection the scheme will perform well. The discretisation gives accurate approximations for smooth solutions, but also steep interior/boundary layers can be represented well. However, we have to exclude discontinuities, since the solution on which the flux is based is assumed to be continuous across a cell interface. Typical applications would be the numerical computation of the detailed structure of a flame front for laminar flames or of a pn-junction in semiconductor devices. Applications in fluid dynamics are restricted to incompressible or weakly compressible flow. We like to emphasise that the method is also suitable to solve pure advection-reaction problems, provided the solution is smooth.

We have organised our paper as follows. The finite volume method is briefly summarised in Sect. 2. In Sect. 3 we derive an integral representation for the flux, in terms of a Green's function, which will be used in Sect. 4 to derive the numerical flux approximation. The combined complete flux-finite volume scheme is presented in Sect. 5. Extension of the method to two-dimensional and time-dependent equations is presented in Sect. 6 and Sect. 7, respectively. To test the scheme, we apply it in Sect. 8 to several model problems. Finally, we end with a summary and conclusions in Sect. 9.

\section{Finite Volume Discretisation}

In this section we outline the FVM for a generic conservation law of advection-diffusionreaction type, defined on a domain in $\mathrm{R}^{d}(d=1,2,3)$. Therefore, consider the following equation

$$
\frac{\partial \varphi}{\partial t}+\nabla \cdot(\boldsymbol{u} \varphi-\varepsilon \nabla \varphi)=s
$$

where $\boldsymbol{u}$ is a velocity or an electric field (flow/drift), $\varepsilon \geq \varepsilon_{\min }>0$ a diffusion/conduction coefficient and $s$ a source term. The unknown $\varphi$ can be, e.g., the temperature or the concentration of a species in a reacting flow. The parameters $\varepsilon$ and $s$ are usually functions of the unknown $\varphi$, however, for the sake of discretisation we will consider these as given functions of the spatial coordinate $\boldsymbol{x}$ and the time $t$. The vector $\boldsymbol{u}$ has to be computed from (flow) equations corresponding to (2.1) and is also considered to be a function of $\boldsymbol{x}$ and $t$. Equations of this type arise, e.g., in combustion theory [21] or plasma physics [23].

Associated with (2.1) we introduce the flux vector $\boldsymbol{f}$, defined by

$$
\boldsymbol{f}:=\boldsymbol{u} \varphi-\varepsilon \nabla \varphi
$$


Fig. 1 A two-dimensional control volume $\Omega_{j}, j=(i, j)$, with its four adjacent cells $\Omega_{\mathrm{k}}$. The circles denote grid points $\boldsymbol{x}_{j}$ and $\boldsymbol{x}_{\mathrm{k}}$; the arrows denote the normal components of the numerical flux $(\boldsymbol{F} \cdot \boldsymbol{n})_{\mathrm{j}, \mathrm{k}}$

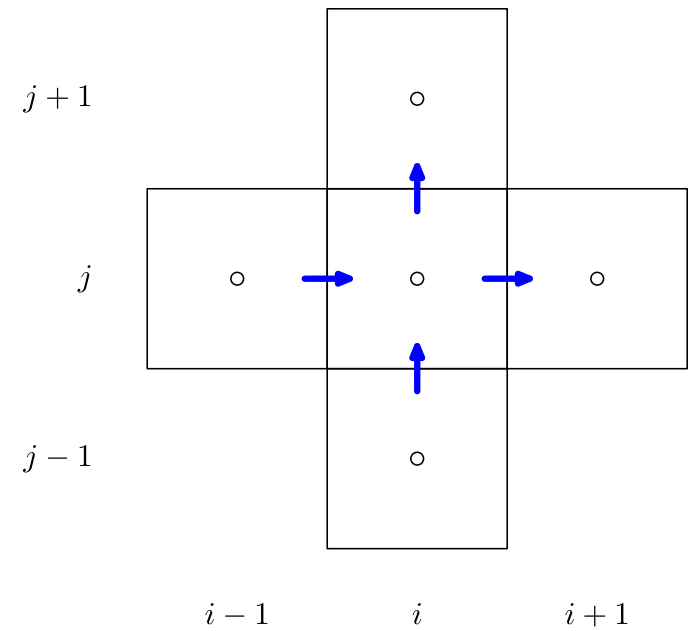

Equation (2.1) then reduces to $\frac{\partial}{\partial t} \varphi+\nabla \cdot f=s$. Integrating this equation over a fixed domain $\Omega \subset \mathrm{R}^{d}$ we obtain the integral form of the conservation law, i.e.,

$$
\frac{\mathrm{d}}{\mathrm{d} t} \int_{\Omega} \varphi \mathrm{d} V+\oint_{\Gamma} \boldsymbol{f} \cdot \boldsymbol{n} \mathrm{d} S=\int_{\Omega} s \mathrm{~d} V
$$

where $\boldsymbol{n}$ is the outward unit normal on the boundary $\Gamma=\partial \Omega$. This equation is the basic conservation law, which reduces to (2.1) provided $\varphi$ is smooth enough.

In the FVM we cover the domain with a finite number of disjunct control volumes or cells $\Omega_{j}$ and impose the integral form (2.3) on each of these cells. The index $j$ is an index vector for multi-dimensional problems. We restrict ourselves to rectangular cells and adopt the cell-centred approach [34], i.e., we choose the grid points $\boldsymbol{x}_{j}$ where the variable $\varphi$ has to be approximated in the cell centres. Consider as an example the two-dimensional cell $\Omega_{j}$ in Fig. 1, for which (2.3) can be written as

$$
\frac{\mathrm{d}}{\mathrm{d} t} \int_{\Omega_{j}} \varphi \mathrm{d} A+\sum_{\mathrm{k} \in \mathcal{N}(j)} \int_{\Gamma_{j, k}} \boldsymbol{f} \cdot \boldsymbol{n} \mathrm{d} s=\int_{\Omega_{j}} s \mathrm{~d} A,
$$

where $\mathcal{N}(j)$ is the index set of neighbouring grid points of $\boldsymbol{x}_{j}$ and where $\Gamma_{j, k}$ is the segment or edge of the boundary $\Gamma_{j}=\partial \Omega_{j}$ connecting the adjacent cells $\Omega_{j}$ and $\Omega_{\mathrm{k}}$. The orientation of $\Gamma_{j}$ is counterclockwise. Approximating all integrals in (2.4) by the midpoint rule, we obtain the following semi-discrete conservation law for $\varphi_{j}(t) \approx \varphi\left(\boldsymbol{x}_{j}, t\right)$, i.e.,

$$
\dot{\varphi}_{j}(t) A_{j}+\sum_{k \in \mathcal{N}(j)}(\boldsymbol{F} \cdot \boldsymbol{n})_{j, k} \ell_{j, k}=s_{j}(t) A_{j},
$$

where $A_{j}$ is the area of $\Omega_{j}, \ell_{j, \mathrm{k}}$ the length of $\Gamma_{j, \mathrm{k}}, \dot{\varphi}_{j}(t) \approx \frac{\partial}{\partial t} \varphi\left(\boldsymbol{x}_{j}, t\right)$ and $s_{j}(t)=s\left(\boldsymbol{x}_{j}, t\right)$. Furthermore, $(\boldsymbol{F} \cdot \boldsymbol{n})_{j, \mathrm{k}}$ is the normal component on $\Gamma_{j, \mathrm{k}}$, directed from $\boldsymbol{x}_{\mathrm{j}}$ to $\boldsymbol{x}_{\mathrm{k}}$, at the interface point $\boldsymbol{x}_{j, \mathrm{k}}:=\frac{1}{2}\left(\boldsymbol{x}_{j}+\boldsymbol{x}_{\mathrm{k}}\right) \in \Gamma_{j, \mathrm{k}}$ of the numerical flux vector $\boldsymbol{F}$, approximating $\boldsymbol{f} \cdot \boldsymbol{n}\left(\boldsymbol{x}_{\mathrm{j}, \mathrm{k}}, t\right)$. Obviously, for stationary problems all time derivatives in (2.4) and (2.5) can be discarded.

The FVM has to be completed with expressions for the numerical flux. We require that $(\boldsymbol{F} \cdot \boldsymbol{n})_{j, k}$ depends on $\varphi$ and a modified source term $\tilde{s}$ in the neighbouring grid points $\boldsymbol{x}_{j}$ and 
$\boldsymbol{x}_{\mathrm{k}}$, i.e., we are looking for an expression of the form

$$
(\boldsymbol{F} \cdot \boldsymbol{n})_{j, k}=\alpha_{j, k} \varphi_{j}-\beta_{j, k} \varphi_{k}+d_{j, k}\left(\gamma_{j, k} \tilde{s}_{j}+\delta_{j, k} \tilde{s}_{k}\right),
$$

where $d_{j, \mathrm{k}}:=\left|\boldsymbol{x}_{j}-\boldsymbol{x}_{\mathrm{k}}\right|$. The variable $\tilde{s}$ includes the source term and additional terms like the cross flux or time derivative, when appropriate. Substitution of (2.6) into (2.5) leads to a linear system for stationary problems or an implicit ODE system for time-dependent problems. The derivation of expressions for the numerical flux is detailed in the next sections.

\section{Integral Representation for the Flux}

In this section we restrict ourselves to one-dimensional steady conservation laws, for which the flux is given by

$$
f=u \varphi-\varepsilon \varphi^{\prime},
$$

where the prime (') denotes differentiation with respect to $x$. Our objective is to derive an integral representation for this flux, based on a Green's function. The derivation is a modification of the theory in [8].

The derivation of the expression for the flux $f_{j+1 / 2}$ at the cell edge $x_{j+1 / 2}=\frac{1}{2}\left(x_{j}+x_{j+1}\right)$ is based on the following model BVP

$$
\begin{aligned}
& \left(u \varphi-\varepsilon \varphi^{\prime}\right)^{\prime}=s, \quad x_{j}<x<x_{j+1}, \\
& \varphi\left(x_{j}\right)=\varphi_{j}, \quad \varphi\left(x_{j+1}\right)=\varphi_{j+1} .
\end{aligned}
$$

We like to emphasise that $f_{j+1 / 2}$ corresponds to the solution of the inhomogeneous BVP (3.2), implying that $f_{j+1 / 2}$ not only depends on $u$ and $\varepsilon$ but on $s$ as well.

In the following, we need the variables $\lambda, P, \Lambda$ and $S$, defined by

$$
\lambda:=\frac{u}{\varepsilon}, \quad P:=\lambda \Delta x, \quad \Lambda(x):=\int_{x_{j+1 / 2}}^{x} \lambda(\xi) \mathrm{d} \xi, \quad S(x):=\int_{x_{j+1 / 2}}^{x} s(\xi) \mathrm{d} \xi,
$$

with $\Delta x:=x_{j+1}-x_{j}$. We refer to the variables $P$ and $\Lambda$ as the (numerical) Peclet function and Peclet integral, respectively, generalising the well-known (numerical/grid) Peclet number [17, 34]. Integrating (3.2a) from $x_{j+1 / 2}$ to $x \in\left(x_{j}, x_{j+1}\right)$ we get the integral balance

$$
f(x)-f_{j+1 / 2}=S(x) .
$$

Using the definition of $\Lambda$ in (3.3), it is clear that expression (3.1) for the flux can be rewritten as

$$
f=-\varepsilon\left(\varphi \mathrm{e}^{-\Lambda}\right)^{\prime} \mathrm{e}^{\Lambda}
$$

Substituting (3.5) in (3.4) and integrating the resulting equation from $x_{j}$ to $x_{j+1}$ we obtain the following expression for the flux $f_{j+1 / 2}$ :

$$
\begin{aligned}
& f_{j+1 / 2}=f_{j+1 / 2}^{\mathrm{h}}+f_{j+1 / 2}^{\mathrm{i}}, \\
& f_{j+1 / 2}^{\mathrm{h}}=-\left(\mathrm{e}^{-\Lambda_{j+1}} \varphi_{j+1}-\mathrm{e}^{-\Lambda_{j}} \varphi_{j}\right) / \int_{x_{j}}^{x_{j+1}} \varepsilon^{-1} \mathrm{e}^{-\Lambda} \mathrm{d} x, \\
& f_{j+1 / 2}^{\mathrm{i}}=-\int_{x_{j}}^{x_{j+1}} \varepsilon^{-1} \mathrm{e}^{-\Lambda} S \mathrm{~d} x / \int_{x_{j}}^{x_{j+1}} \varepsilon^{-1} \mathrm{e}^{-\Lambda} \mathrm{d} x,
\end{aligned}
$$



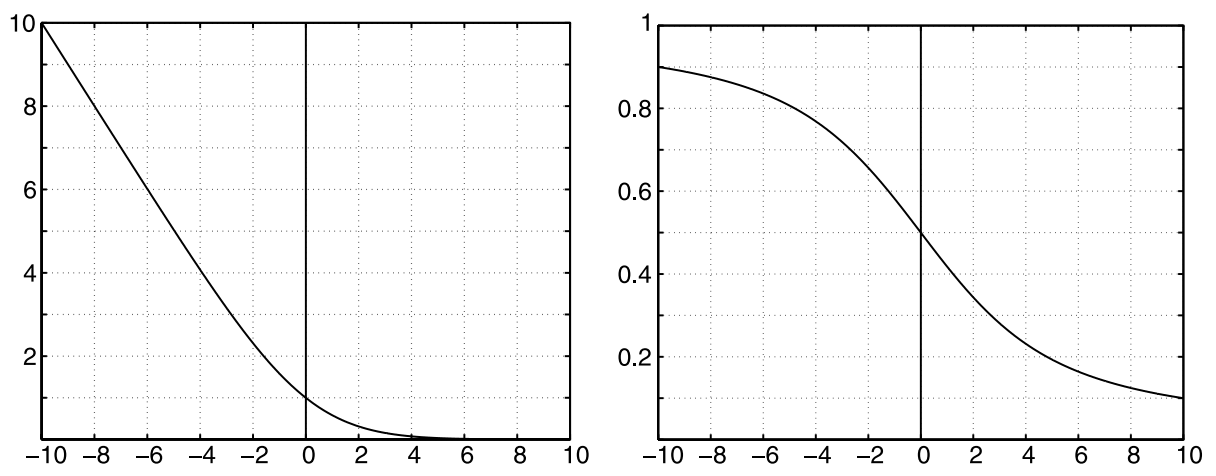

Fig. 2 The Bernoulli function $B$ (left) and the function $W$ (right)

where $f_{j+1 / 2}^{\mathrm{h}}$ and $f_{j+1 / 2}^{\mathrm{i}}$ are the homogeneous and inhomogeneous part, corresponding to the homogeneous and particular solution of (3.2), respectively.

Assume first that $u, \varepsilon$ and $s$ are constant on the interval $\left[x_{j}, x_{j+1}\right]$. In this case we can determine all integrals in (3.3). The Peclet function reduces to the Peclet number, i.e., $P=$ $u \Delta x / \varepsilon$. Furthermore, $\Lambda(x)=\lambda\left(x-x_{j+1 / 2}\right)$ and $S(x)=s\left(x-x_{j+1 / 2}\right)$. Substituting these expressions in (3.6b) and (3.6c) and evaluating all integrals involved, we find

$$
\begin{aligned}
f_{j+1 / 2}^{\mathrm{h}} & =-\frac{\varepsilon}{\Delta x}\left(B(P) \varphi_{j+1}-B(-P) \varphi_{j}\right), \\
f_{j+1 / 2}^{\mathrm{i}} & =\left(\frac{1}{2}-W(P)\right) s \Delta x
\end{aligned}
$$

where we have introduced the functions $B$ and $W$, defined by

$$
B(z):=\frac{z}{\mathrm{e}^{z}-1}, \quad W(z):=\frac{\mathrm{e}^{z}-1-z}{z\left(\mathrm{e}^{z}-1\right)} ;
$$

see Fig. 2. The function $B$ is the generating function of the Bernoulli numbers [28], in short referred to as the Bernoulli function. Note that $W$ satisfies $0 \leq W(z) \leq 1$ and $W(-z)+$ $W(z)=1$. Clearly, the inhomogeneous flux $f_{j+1 / 2}^{\mathrm{i}}$ is of importance when $|P| \gg 1$, i.e., for advection dominated flow. For the constant coefficient homogeneous flux we introduce the function

$$
f_{j+1 / 2}^{\mathrm{h}}=\mathcal{F}^{\mathrm{h}}\left(\varepsilon / \Delta x, P ; \varphi_{j}, \varphi_{j+1}\right)=\alpha_{j+1 / 2}(\varepsilon / \Delta x, P) \varphi_{j}-\beta_{j+1 / 2}(\varepsilon / \Delta x, P) \varphi_{j+1},
$$

to denote the dependence of $f_{j+1 / 2}^{\mathrm{h}}$ on the parameters $\varepsilon / \Delta x$ and $P$ and on the function values $\varphi_{j}$ and $\varphi_{j+1}$; cf. (2.6). The constant coefficient homogeneous flux is often used as approximation of the flux (2.2); see, e.g., [20].

We will next generalise the constant coefficient fluxes (3.7a) and (3.7b) for the case of variable $u, \varepsilon$ and $s$. Let $\langle a, b\rangle$ denote the usual inner product of two functions $a=a(x)$ and $b=b(x)$ defined on $\left(x_{j}, x_{j+1}\right)$, i.e.,

$$
\langle a, b\rangle:=\int_{x_{j}}^{x_{j+1}} a(x) b(x) \mathrm{d} x .
$$


Introducing the average $\bar{\Lambda}_{j+1 / 2}:=\frac{1}{2}\left(\Lambda_{j}+\Lambda_{j+1}\right)$ and using the relation $\Lambda_{j+1}-\Lambda_{j}=\langle\lambda, 1\rangle$, we can rewrite the expression (3.6b) for the homogeneous flux as

$$
f_{j+1 / 2}^{\mathrm{h}}=-\mathrm{e}^{-\bar{\Lambda}_{j+1 / 2}}\left(\mathrm{e}^{-\langle\lambda, 1\rangle / 2} \varphi_{j+1}-\mathrm{e}^{\langle\lambda, 1\rangle / 2} \varphi_{j}\right) /\left\langle\varepsilon^{-1}, \mathrm{e}^{-\Lambda}\right\rangle .
$$

It is even possible to formulate this expression as a modification of the constant coefficient homogeneous flux (3.7a), in the following way

$$
f_{j+1 / 2}^{\mathrm{h}}=\mathcal{F}^{\mathrm{h}}\left(\frac{\left\langle\lambda, \mathrm{e}^{-\Lambda}\right\rangle /\langle\lambda, 1\rangle}{\left\langle\varepsilon^{-1}, \mathrm{e}^{-\Lambda}\right\rangle},\langle\lambda, 1\rangle ; \varphi_{j}, \varphi_{j+1}\right) .
$$

Our numerical approximation of the homogeneous flux will be based on (3.12).

The inhomogeneous flux can be written as a weighted average of the variable $S$ as follows:

$$
f_{j+1 / 2}^{\mathrm{i}}=-\frac{\left\langle\varepsilon^{-1} S, \mathrm{e}^{-\Lambda}\right\rangle}{\left\langle\varepsilon^{-1}, \mathrm{e}^{-\Lambda}\right\rangle} .
$$

Substituting the expression for $S$ in (3.6c) and changing the order of integration we find the following alternative representation for the inhomogeneous flux

$$
f_{j+1 / 2}^{\mathrm{i}}=\Delta x \int_{0}^{1} G(\sigma) s(x(\sigma)) \mathrm{d} \sigma, \quad \sigma(x):=\frac{x-x_{j}}{\Delta x},
$$

where $\sigma=\sigma(x)$ is the normalised coordinate on $\left[x_{j}, x_{j+1}\right]$ and $x=x(\sigma)$ its inverse, and where $G(\sigma)$ is the Green's function for the flux, given by

$$
G(\sigma)= \begin{cases}\Delta x \int_{0}^{\sigma} \varepsilon^{-1}(x(\eta)) \mathrm{e}^{-\Lambda(x(\eta))} \mathrm{d} \eta /\left\langle\varepsilon^{-1}, \mathrm{e}^{-\Lambda}\right\rangle & \text { for } 0 \leq \sigma \leq \frac{1}{2}, \\ -\Delta x \int_{\sigma}^{1} \varepsilon^{-1}(x(\eta)) \mathrm{e}^{-\Lambda(x(\eta))} \mathrm{d} \eta /\left\langle\varepsilon^{-1}, \mathrm{e}^{-\Lambda}\right\rangle & \text { for } \frac{1}{2}<\sigma \leq 1,\end{cases}
$$

with $x(\eta):=x_{j}+\eta \Delta x$. Note that $G$ relates the $f l u x$ to the source term and is different from the usual Green's function, which relates the solution to the source term; see e.g. [17]. For the special case of constant $u$ and $\varepsilon$ this Green's function reduces to

$$
G(\sigma ; P)= \begin{cases}\frac{1-\mathrm{e}^{-P \sigma}}{1-\mathrm{e}^{-P}} & \text { for } 0 \leq \sigma \leq \frac{1}{2} \\ -\frac{1-\mathrm{e}^{P(1-\sigma)}}{1-\mathrm{e}^{P}} & \text { for } \frac{1}{2}<\sigma \leq 1\end{cases}
$$

see Fig. 3. Note that we use the notation $G=G(\sigma ; P)$ to denote the dependence on the numerical Peclet number $P$. For constant $s$ we can evaluate the integral in (3.14) and recover the constant coefficient flux (3.7b).

The Green's function (3.16) for the flux has the following properties. First, it is discontinuous at $\sigma=\frac{1}{2}$, corresponding to $x=x_{j+1 / 2}$, with jump $G\left(\frac{1}{2}-; P\right)-G\left(\frac{1}{2}+; P\right)=1$. Second, for $|P| \gg 1$, the average value on the half interval upwind of $\sigma=\frac{1}{2}$, i.e., the interval $\left[0, \frac{1}{2}\right]$ for $u \geq 0$ and $\left[\frac{1}{2}, 1\right]$ for $u<0$, is much larger than the average on the downwind half, which means that for dominant advection the upwind value of the source term is the relevant one. On the other hand, for dominant diffusion, i.e., $|P|$ is small, the average value $\frac{1}{2}-W(P)$ is close to 0 , implying that the inhomogeneous flux is not important. Finally, it satisfies the symmetry property $G(\sigma ; P)=-G(1-\sigma ;-P)$. 

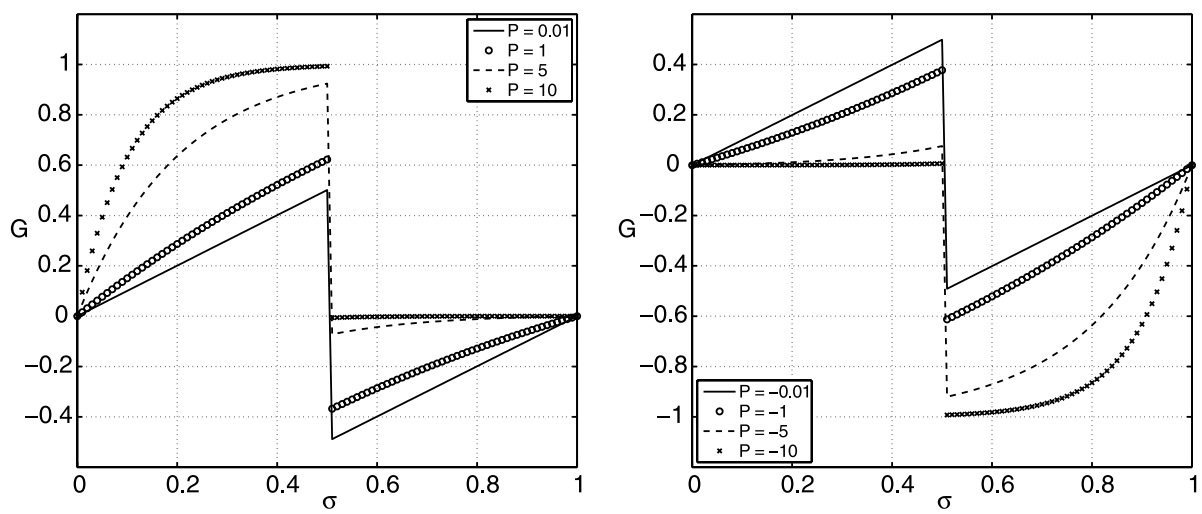

Fig. 3 Green's function for the flux for $P>0$ (left) and $P<0$ (right)

When only $u(x)=$ Const $\neq 0$ on $\left[x_{j}, x_{j+1}\right]$, the expression (3.14) for the inhomogeneous flux can be written as

$$
f_{j+1 / 2}^{\mathrm{i}}=\Delta x \int_{0}^{1} G(\sigma ;\langle\lambda, 1\rangle) s(x(\sigma)) \mathrm{d} \sigma
$$

with $G(\sigma ; P)$ the constant coefficient Green's function defined in (3.16) and where $\sigma$ is a weighted normalised coordinate defined by

$$
\sigma(x):=\int_{x_{\mathrm{C}}}^{x} \lambda(\xi) \mathrm{d} \xi /\langle\lambda, 1\rangle .
$$

Note that $\sigma^{\prime}>0$ implying that $\sigma$ is monotonically increasing from 0 to 1 indeed.

To summarise, the flux $f_{j+1 / 2}$ is the superposition (3.6a) of the homogeneous flux $f_{j+1 / 2}^{\mathrm{h}}$, given in (3.12), and the inhomogeneous flux $f_{j+1 / 2}^{\mathrm{i}}$. For the latter flux we approximate $u(x)$ on $\left[x_{j}, x_{j+1}\right]$ by a constant and employ the representation (3.17), with $G(\sigma ; P)$ defined in (3.16).

\section{Derivation of the Numerical Flux}

In this section we give quadrature rules for the inner products $\langle\lambda, 1\rangle$ and $\left\langle a, \mathrm{e}^{-\Lambda}\right\rangle, \quad(a=$ $\left.\lambda, \varepsilon^{-1}\right)$. This readily gives an approximation of (3.12). Moreover, we propose an approximation for the integral in (3.17). Our objective is to obtain a numerical flux approximation that is second order accurate, uniformly in the local Peclet numbers.

First, we introduce the average $\bar{a}_{j+1 / 2}$, the weighted average $\tilde{a}_{j+1 / 2}$ and the upwind value $a_{\mathrm{u}, j+1 / 2}$ of a variable $a=a(x)$ as follows

$$
\begin{aligned}
& \bar{a}_{j+1 / 2}:=\frac{1}{2}\left(a_{j}+a_{j+1}\right), \\
& \tilde{a}_{j+1 / 2}:=W\left(-\bar{P}_{j+1 / 2}\right) a_{j}+W\left(\bar{P}_{j+1 / 2}\right) a_{j+1}, \\
& a_{\mathrm{u}, j+1 / 2}:= \begin{cases}a_{j} & \text { if } \bar{u}_{j+1 / 2} \geq 0, \\
a_{j+1} & \text { if } \bar{u}_{j+1 / 2}<0 .\end{cases}
\end{aligned}
$$


The weights in the expression for $\tilde{a}_{j+1 / 2}$ are determined by the average Peclet number $\bar{P}_{j+1 / 2}$. Note that the weighted average $\tilde{a}_{j+1 / 2}$ reduces to the ordinary average $\bar{a}_{j+1 / 2}$ for $\bar{P}_{j+1 / 2} \rightarrow 0$ and to $a_{\mathrm{u}, j+1 / 2}$ for $\left|\bar{P}_{j+1 / 2}\right| \rightarrow \infty$. This is also apparent from the following relation

$$
\tilde{a}_{j+1 / 2}=2 W\left(\left|\bar{P}_{j+1 / 2}\right|\right) \bar{a}_{j+1 / 2}+\left(1-2 W\left(\left|\bar{P}_{j+1 / 2}\right|\right)\right) a_{\mathrm{u}, j+1 / 2},
$$

which can be readily verified from (4.1). In the derivation of the numerical flux that follows, we need the 'product rule'

$$
\tilde{a}_{j+1 / 2} \tilde{b}_{j+1 / 2}=(\widetilde{a b})_{j+1 / 2}-W\left(\bar{P}_{j+1 / 2}\right) W\left(-\bar{P}_{j+1 / 2}\right)\left(a_{j+1}-a_{j}\right)\left(b_{j+1}-b_{j}\right) .
$$

A similar rule for $\bar{a}_{j+1 / 2}$ can be easily derived substituting $\bar{P}_{j+1 / 2}=0$ in (4.3).

For the inner product $\langle\lambda, 1\rangle$ we use the standard trapezoidal rule, which can be written as

$$
\langle\lambda, 1\rangle=\bar{P}_{j+1 / 2}-\frac{1}{12} \lambda^{\prime \prime}(\xi) \Delta x^{3}, \quad \xi \in\left(x_{j}, x_{j+1}\right) .
$$

In the derivation of the trapezoidal rule (4.4) we have replaced $\lambda$ by its linear interpolant on $\left[x_{j}, x_{j+1}\right]$, however, this is not a suitable approach for the inner products $\left\langle a, \mathrm{e}^{-\Lambda}\right\rangle$. Instead, we approximate both $a$ and $\Lambda$ by their linear interpolants, resulting in the following generalised trapezoidal rule

$$
\frac{\left\langle a, \mathrm{e}^{-\Lambda}\right\rangle}{\left\langle 1, \mathrm{e}^{-\Lambda}\right\rangle}=\tilde{a}_{j+1 / 2}+E_{j+1 / 2}(a), \quad\left|E_{j+1 / 2}(a)\right|<C \Delta x^{2},
$$

for some $C>0$, which holds provided $a$ is twice and $P$ once continuously differentiable on $\left(x_{j}, x_{j+1}\right)$. For a proof of this rule see [8].

For the homogeneous flux (3.12) we need to evaluate the first argument of $\mathcal{F}^{\mathrm{h}}$. Applying the quadrature rules (4.4), (4.5) and the product rule (4.3), with $a=\varepsilon$ and $b=\varepsilon^{-1}$, we can derive the following second order approximation

$$
\frac{\left\langle\lambda, \mathrm{e}^{-\Lambda}\right\rangle /\langle\lambda, 1\rangle}{\left\langle\varepsilon^{-1}, \mathrm{e}^{-\Lambda}\right\rangle} \doteq \frac{1}{\bar{P}_{j+1 / 2}} \frac{\tilde{\lambda}_{j+1 / 2}}{\widetilde{\left(\varepsilon^{-1}\right)_{j+1 / 2}}} \doteq \frac{\tilde{\lambda}_{j+1 / 2}}{\bar{\lambda}_{j+1 / 2}} \frac{\tilde{\varepsilon}_{j+1 / 2}}{\Delta x}
$$

Note that $\tilde{\lambda}_{j+1 / 2} / \bar{\lambda}_{j+1 / 2} \rightarrow 1$ and $\tilde{\varepsilon}_{j+1 / 2} \rightarrow \bar{\varepsilon}_{j+1 / 2}$ for $\bar{P}_{j+1 / 2} \rightarrow 0$; cf. (4.2). Substituting this expression in (3.12) we obtain the homogeneous numerical flux

$$
F_{j+1 / 2}^{\mathrm{h}}=\mathcal{F}^{\mathrm{h}}\left(\frac{\mathcal{E}_{j+1 / 2}}{\Delta x}, \bar{P}_{j+1 / 2} ; \varphi_{j}, \varphi_{j+1}\right), \quad \mathcal{E}_{j+1 / 2}:=\frac{\tilde{\lambda}_{j+1 / 2}}{\bar{\lambda}_{j+1 / 2}} \tilde{\varepsilon}_{j+1 / 2},
$$

which is in fact the constant coefficient flux defined in (3.7a) and (3.9), with $\varepsilon$ and $P$ replaced by $\mathcal{E}_{j+1 / 2}$ and $\bar{P}_{j+1 / 2}$, respectively.

For the inhomogeneous flux we note that the Green's function $G(\sigma ; P)$ has a clear bias towards the upwind side of the interval when $|P| \gg 1$. For that reason we replace $s(x(\sigma))$ in (3.17) by its upwind value and evaluate the resulting integral exactly. This way we obtain for the inhomogeneous numerical flux

$$
F_{j+1 / 2}^{\mathrm{i}}=\left(\frac{1}{2}-W\left(\bar{P}_{j+1 / 2}\right)\right) s_{\mathrm{u}, j+1 / 2} \Delta x,
$$


which is the constant coefficient flux (3.7b) with $P$ and $s$ replaced by $\bar{P}_{j+1 / 2}$ and $s_{\mathrm{u}, j+1 / 2}$, respectively.

The final numerical flux $F_{j+1 / 2}$ is the superposition of the homogeneous part $F_{j+1 / 2}^{\mathrm{h}}$ and the inhomogeneous part $F_{j+1 / 2}^{\mathrm{i}}$, i.e.,

$$
F_{j+1 / 2}=F_{j+1 / 2}^{\mathrm{h}}+F_{j+1 / 2}^{\mathrm{i}},
$$

with $F_{j+1 / 2}^{\mathrm{h}}$ and $F_{j+1 / 2}^{\mathrm{i}}$ given in (4.7) and (4.8), respectively; see also [8]. We refer to the flux approximation in (4.7)-(4.9) as the complete flux (CF) scheme.

\section{The Finite Volume-Complete Flux Scheme}

To derive the final scheme, we combine the complete flux approximation in (4.7)-(4.9) with the discrete conservation law for (3.2a), given by

$$
F_{j+1 / 2}-F_{j-1 / 2}=s_{j} \Delta x
$$

cf. (2.5). The numerical flux at the cell interface $x_{j+1 / 2}$ can be written as

$$
F_{j+1 / 2}=\alpha_{j+1 / 2} \varphi_{j}-\beta_{j+1 / 2} \varphi_{j+1}+\Delta x\left(\gamma_{j+1 / 2} s_{j}+\delta_{j+1 / 2} s_{j+1}\right),
$$

where the coefficients $\alpha_{j+1 / 2}, \beta_{j+1 / 2}$ etc. are defined by

$$
\begin{aligned}
\alpha_{j+1 / 2} & :=\frac{\mathcal{E}_{j+1 / 2}}{\Delta x} B_{j+1 / 2}^{-}, \quad \beta_{j+1 / 2}:=\frac{\mathcal{E}_{j+1 / 2}}{\Delta x} B_{j+1 / 2}^{+}, \quad B_{j+1 / 2}^{ \pm}:=B\left( \pm \bar{P}_{j+1 / 2}\right), \\
\gamma_{j+1 / 2} & =\max \left(\frac{1}{2}-W_{j+1 / 2}^{+}, 0\right), \quad \delta_{j+1 / 2}=\min \left(\frac{1}{2}-W_{j+1 / 2}^{+}, 0\right) \\
W_{j+1 / 2}^{+} & :=W\left(\bar{P}_{j+1 / 2}\right),
\end{aligned}
$$

cf. (2.6). The formulae for $\gamma_{j+1 / 2}$ and $\delta_{j+1 / 2}$ hold provided the grid size is small enough such that $\operatorname{sgn}\left(\bar{u}_{j+1 / 2}\right)=\operatorname{sgn}\left(\bar{P}_{j+1 / 2}\right)$, which we henceforth assume. A similar expression holds for the numerical flux $F_{j-1 / 2}$ at the cell interface $x_{j-1 / 2}$. Substituting these in the discrete conservation law (5.1) we obtain

$$
-a_{\mathrm{W}, j} \varphi_{j-1}+a_{\mathrm{C}, j} \varphi_{j}-a_{\mathrm{E}, j} \varphi_{j+1}=b_{\mathrm{W}, j} s_{j-1}+b_{\mathrm{C}, j} s_{j}+b_{\mathrm{E}, j} s_{j+1},
$$

referred to as the finite volume-complete flux (FV-CF) scheme, with the coefficients $a_{\mathrm{W}, j}$, $b_{\mathrm{W}, j}$ etc. defined by

$$
\begin{aligned}
& a_{\mathrm{W}, j}:=\alpha_{j-1 / 2}, \quad a_{E, j}:=\beta_{j+1 / 2}, \quad a_{C, j}:=\alpha_{j+1 / 2}+\beta_{j-1 / 2}, \\
& b_{\mathrm{W}, j}:=\gamma_{j-1 / 2} \Delta x, \quad b_{\mathrm{E}, j}:=-\delta_{j+1 / 2} \Delta x, \quad b_{\mathrm{C}, j}=\left(1-\gamma_{j+1 / 2}+\delta_{j-1 / 2}\right) \Delta x .
\end{aligned}
$$

Note that $b_{\mathrm{W}, j}, b_{\mathrm{E}, j}, b_{\mathrm{C}, j} \geq 0$ and $b_{\mathrm{W}, j}+b_{\mathrm{C}, j}+b_{\mathrm{E}, j}=\left(1+W_{j+1 / 2}^{+}-W_{j-1 / 2}^{+}\right) \Delta x$. The FV$\mathrm{CF}$ scheme has a three-point coupling for both $\varphi$ and $s$, resulting in the following linear system

$$
A \varphi=B s+b,
$$


where $\varphi$ and $s$ are the vector of unknowns and source terms, respectively, and where the vector $\boldsymbol{b}$ contains the boundary data. Both matrices $\boldsymbol{A}$ and $\boldsymbol{B}$ are tridiagonal. For the special case of constant $u$ and $\varepsilon$, we can easily prove that $a_{\mathrm{W}, j}, a_{\mathrm{E}, j} \geq 0$ and $a_{\mathrm{C}, j}=a_{\mathrm{W}, j}+a_{\mathrm{E}, j}$, and as a consequence the matrix $\boldsymbol{A}$ is an M-matrix, provided not both boundary conditions are of Neumann type.

In our numerical examples in Sect. 8 we compare the CF scheme for the flux approximation with the homogeneous flux (HF) scheme, which only includes the homogeneous component (4.7). This means that $\gamma_{j+1 / 2}=\delta_{j+1 / 2}=0$ in (5.2a) and hence $b_{\mathrm{W}, j}=b_{\mathrm{E}, j}=0$ and $b_{\mathrm{C}, j}=\Delta x$ in (5.3).

It is instructive to consider some limiting cases of the FV-CF scheme. First, we take $u=0$, i.e., we consider the equation $-\left(\varepsilon \varphi^{\prime}\right)^{\prime}=s$. In this case $\bar{P}_{j \pm 1 / 2}=0$ and consequently the inhomogeneous fluxes vanish, resulting in the second order central difference scheme

$$
-\frac{1}{\Delta x}\left(\bar{\varepsilon}_{j+1 / 2}\left(\varphi_{j+1}-\varphi_{j}\right)-\bar{\varepsilon}_{j-1 / 2}\left(\varphi_{j}-\varphi_{j-1}\right)\right)=s_{j} \Delta x .
$$

Another limiting case is $\varepsilon=0$, corresponding to the reduced equation $(u \varphi)^{\prime}=s$. For this equation we have to distinguish between $u>0$ and $u<0$. In the former case, $\bar{P}_{j \pm 1 / 2} \rightarrow+\infty$ and the FV-CF scheme (5.3) reduces to

$$
u_{j} \varphi_{j}-u_{j-1} \varphi_{j-1}=\frac{1}{2}\left(s_{j-1}+s_{j}\right) \Delta x
$$

In the latter case, $\bar{P}_{j \pm 1 / 2} \rightarrow-\infty$, giving the scheme

$$
u_{j+1} \varphi_{j+1}-u_{j} \varphi_{j}=\frac{1}{2}\left(s_{j}+s_{j+1}\right) \Delta x
$$

Both schemes in (5.7) can be interpreted a second order cell-vertex FVM [18] with the control volumes moved over a distance $\frac{1}{2} \Delta x$ in the upwind direction. Here we see why it is important that our flux approximation includes the source term. Standard methods like the HF scheme omit the inhomogeneous flux, so that the schemes in (5.7) further reduce to $u_{j} \varphi_{j}-u_{j-1} \varphi_{j-1}=s_{j} \Delta x$ for $u>0$ or $u_{j+1} \varphi_{j+1}-u_{j} \varphi_{j}=s_{j} \Delta x$ for $u<0$, which is just the first order upwind scheme for the reduced advection-reaction equation.

From these observations we conclude that the FV-CF scheme (5.3) can be interpreted as a combination of the central difference scheme (5.6) and the schemes (5.7), the combination determined by the (average) Peclet numbers $\bar{P}_{j \pm 1 / 2}$.

\section{Extension to Two-dimensional Conservation Laws}

In this section we extend the derivation to two-dimensional steady conservation laws. In particular, we derive the expression for the $x$-component of the numerical flux (the derivation of the $y$-component is similar) and present the final scheme. For ease of notation, we use the compass notation; see Fig. 4. Thus, $\varphi_{\mathrm{C}}$ should be understood as $\varphi_{i, j}, f_{\mathrm{e}}$ as $f_{i+1 / 2, j}$ etc.

The flux corresponding to (2.1) is given by

$$
\boldsymbol{f}=f_{1} \boldsymbol{e}_{x}+f_{2} \boldsymbol{e}_{y}=\left(u \varphi-\varepsilon \frac{\partial \varphi}{\partial x}\right) \boldsymbol{e}_{x}+\left(v \varphi-\varepsilon \frac{\partial \varphi}{\partial y}\right) \boldsymbol{e}_{y} .
$$

We outline the derivation of the $x$-component of the numerical flux $F_{1, \mathrm{e}}$ at the eastern edge of the control volume $\Omega_{\mathrm{C}}$; see Fig. 4 . The derivation of the $y$-component $F_{2, \mathrm{n}}$ of the numerical 
Fig. 4 Control volume $\Omega_{\mathrm{C}}$ and corresponding stencil using compass notation

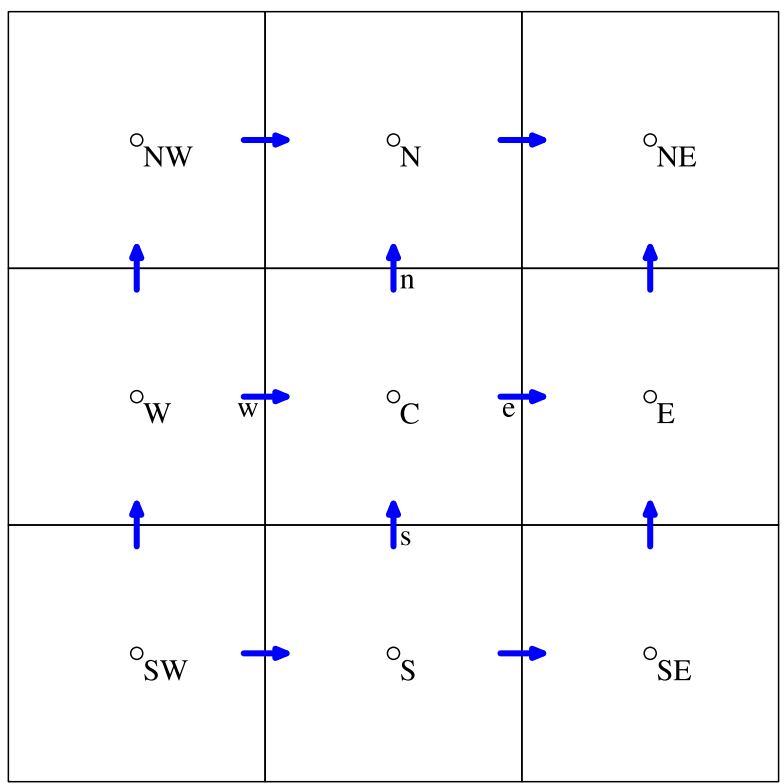

flux at the northern edge is completely analogous and is therefore omitted. Analogous to the derivation in Sect. 3 , the numerical flux $F_{1, \mathrm{e}}$ follows from the quasi-one-dimensional BVP

$$
\begin{aligned}
& \frac{\partial}{\partial x}\left(u \varphi-\varepsilon \frac{\partial \varphi}{\partial x}\right)=s_{x}, \quad x_{\mathrm{C}}<x<x_{\mathrm{E}}, y=y_{\mathrm{e}} \\
& \varphi\left(\boldsymbol{x}_{\mathrm{C}}\right)=\varphi_{\mathrm{C}}, \quad \varphi\left(\boldsymbol{x}_{\mathrm{E}}\right)=\varphi_{\mathrm{E}}
\end{aligned}
$$

where the modified source term $s_{x}$ is defined by

$$
s_{x}:=s-\frac{\partial f_{2}}{\partial y}
$$

The derivation of the expression for the numerical flux is essentially the same as in the one-dimensional case, the main difference being the inclusion of the cross flux term $\partial f_{2} / \partial y$ in the source term. In the computation of $s_{x}$ we replace $\partial f_{2} / \partial y$ by its central difference approximation and for $f_{2}$ we take the homogeneous numerical flux. A similar procedure applies to the $y$-component of the flux. Putting everything together, we obtain the twodimensional complete flux scheme on the next page.

The stencil of the flux approximation for $F_{1, \mathrm{e}}$ is depicted in Fig. 4. Assume first that $\bar{u}_{\mathrm{e}}>0$. Then $F_{1, \mathrm{e}}$ depends on $\varphi$ in the grid points $\boldsymbol{x}_{\mathrm{C}}$ and $\boldsymbol{x}_{\mathrm{E}}$, on $s$ in the central point $\boldsymbol{x}_{\mathrm{C}}$ and on the homogeneous fluxes $F_{2, \mathrm{n}}^{\mathrm{h}}$ and $F_{2, \mathrm{~s}}^{\mathrm{h}}$ and through these fluxes again on $\varphi$ in $\boldsymbol{x}_{\mathrm{N}}$ and $\boldsymbol{x}_{\mathrm{S}}$. For $\bar{u}_{\mathrm{e}}<0, F_{1, \mathrm{e}}$ again depends on $\varphi_{\mathrm{C}}$ and $\varphi_{\mathrm{E}}$, but this time on the source term $s_{\mathrm{E}}$ and the homogeneous fluxes $F_{2, \text { En }}^{\mathrm{h}}$ and $F_{2 \text {,Es }}^{\mathrm{h}}$, inducing a further dependency on $\varphi_{\mathrm{NE}}$ and $\varphi_{\mathrm{SE}}$. Thus, in addition to the direct neighbours, $F_{1, \mathrm{e}}$ depends on a few other values of $\varphi$, determined by the local upwind direction; cf. (2.6). 


\section{Two-dimensional CF scheme}

Peclet function

$P_{1}:=u \Delta x / \varepsilon$

$$
P_{2}:=v \Delta y / \varepsilon
$$

(Weighted) average

$$
\begin{aligned}
& \bar{a}_{\mathrm{e}}:=\frac{1}{2}\left(a_{\mathrm{C}}+a_{\mathrm{E}}\right) \\
& \tilde{a}_{\mathrm{e}}:=W\left(-\bar{P}_{1, \mathrm{e}}\right) a_{\mathrm{C}}+W\left(\bar{P}_{1, \mathrm{e}}\right) a_{\mathrm{E}}
\end{aligned}
$$

$$
\begin{aligned}
& \bar{a}_{\mathrm{n}}:=\frac{1}{2}\left(a_{\mathrm{C}}+a_{\mathrm{N}}\right) \\
& \tilde{a}_{\mathrm{n}}:=W\left(-\bar{P}_{2, \mathrm{n}}\right) a_{\mathrm{C}}+W\left(\bar{P}_{2, \mathrm{n}}\right) a_{\mathrm{N}}
\end{aligned}
$$

Homogeneous flux

$$
F_{1, \mathrm{e}}^{\mathrm{h}}=\mathcal{F}^{\mathrm{h}}\left(\frac{\tilde{P}_{1, \mathrm{e}}}{\bar{P}_{1, \mathrm{e}}} \frac{\tilde{\varepsilon}_{\mathrm{e}}}{\Delta x}, \bar{P}_{1, \mathrm{e}} ; \varphi_{\mathrm{C}}, \varphi_{\mathrm{E}}\right)
$$

$$
F_{2, \mathrm{n}}^{\mathrm{h}}=\mathcal{F}^{\mathrm{h}}\left(\frac{\tilde{P}_{2, \mathrm{n}}}{\bar{P}_{2, \mathrm{n}}} \frac{\tilde{\varepsilon}_{\mathrm{n}}}{\Delta y}, \bar{P}_{2, \mathrm{n}} ; \varphi_{\mathrm{C}}, \varphi_{\mathrm{N}}\right)
$$

Source term with cross wind diffusion

$$
s_{x, \mathrm{C}}=s_{\mathrm{C}}-\frac{1}{\Delta y}\left(F_{2, \mathrm{n}}^{\mathrm{h}}-F_{2, \mathrm{~s}}^{\mathrm{h}}\right)
$$$$
s_{y, \mathrm{C}}=s_{\mathrm{C}}-\frac{1}{\Delta x}\left(F_{1, \mathrm{e}}^{\mathrm{h}}-F_{1, \mathrm{w}}^{\mathrm{h}}\right)
$$

Upwinded source term

$s_{x, \mathrm{u}, \mathrm{e}}= \begin{cases}s_{x, \mathrm{C}} & \text { if } \bar{u}_{\mathrm{e}} \geq 0 \\ s_{x, \mathrm{E}} & \text { if } \bar{u}_{\mathrm{e}}<0\end{cases}$

$s_{y, \mathrm{u}, \mathrm{n}}= \begin{cases}s_{y, \mathrm{C}} & \text { if } \bar{v}_{\mathrm{n}} \geq 0 \\ s_{y, \mathrm{~N}} & \text { if } \bar{v}_{\mathrm{n}}<0\end{cases}$

Inhomogeneous flux

$F_{1, \mathrm{e}}^{\mathrm{i}}=\left(\frac{1}{2}-W\left(\bar{P}_{1, \mathrm{e}}\right)\right) s_{x, \mathrm{u}, \mathrm{e}} \Delta x$

$$
F_{2, \mathrm{n}}^{\mathrm{i}}=\left(\frac{1}{2}-W\left(\bar{P}_{2, \mathrm{n}}\right)\right) s_{y, \mathrm{u}, \mathrm{n}} \Delta y
$$

Complete flux

$$
\underline{F_{1, \mathrm{e}}=F_{1, \mathrm{e}}^{\mathrm{h}}+F_{1, \mathrm{e}}^{\mathrm{i}} \quad F_{2, \mathrm{n}}=F_{2, \mathrm{n}}^{\mathrm{h}}+F_{2, \mathrm{n}}^{\mathrm{i}}}
$$

Next, we formulate the discretisation scheme based on this flux approximation. Introducing flux differences like

$$
\delta_{x} F_{1, \mathrm{C}}:=\frac{1}{\Delta x}\left(F_{1, \mathrm{e}}-F_{1, \mathrm{w}}\right), \quad \delta_{y} F_{2, \mathrm{C}}:=\frac{1}{\Delta y}\left(F_{2, \mathrm{n}}-F_{2, \mathrm{~s}}\right),
$$

it is clear that the discrete conservation law (2.5) can be written as

$$
\delta_{x} F_{1, \mathrm{C}}+\delta_{y} F_{2, \mathrm{C}}=s_{\mathrm{C}} .
$$

All numerical fluxes in (6.4) contain a difference of a homogeneous cross flux. Therefore, substituting the numerical fluxes defined above in (6.4) we obtain the discretisation

$$
\begin{aligned}
\gamma_{2, \mathrm{~s}} & \delta_{x} F_{1, \mathrm{~S}}^{\mathrm{h}}+\left(1-\gamma_{2, \mathrm{n}}+\delta_{2, \mathrm{~s}}\right) \delta_{x} F_{1, \mathrm{C}}^{\mathrm{h}}-\delta_{2, \mathrm{n}} \delta_{x} F_{1, \mathrm{~N}}^{\mathrm{h}} \\
& +\gamma_{1, \mathrm{w}} \delta_{y} F_{2, \mathrm{~W}}^{\mathrm{h}}+\left(1-\gamma_{1, \mathrm{e}}+\delta_{1, \mathrm{w}}\right) \delta_{y} F_{2, \mathrm{C}}^{\mathrm{h}}-\delta_{1, \mathrm{e}} \delta_{y} F_{2, \mathrm{E}}^{\mathrm{h}} \\
= & \left(1-\gamma_{2, \mathrm{n}}+\delta_{2, \mathrm{~s}}-\gamma_{1, \mathrm{e}}+\delta_{1, \mathrm{w}}\right) s_{\mathrm{C}}+\gamma_{2, \mathrm{~s}} s_{\mathrm{S}}-\delta_{2, \mathrm{n}} s_{\mathrm{N}}+\gamma_{1, \mathrm{w}} s_{\mathrm{W}}-\delta_{1, \mathrm{e}} s_{\mathrm{E}},
\end{aligned}
$$

where the coefficients $\gamma_{2, \mathrm{~s}}$ etc. are defined by

$$
\begin{array}{ll}
\gamma_{1, \mathrm{e}}:=\max \left(\frac{1}{2}-W\left(\bar{P}_{1, \mathrm{e}}\right), 0\right), & \delta_{1, \mathrm{e}}=\min \left(\frac{1}{2}-W\left(\bar{P}_{1, \mathrm{e}}\right), 0\right), \\
\gamma_{2, \mathrm{n}}:=\max \left(\frac{1}{2}-W\left(\bar{P}_{2, \mathrm{n}}\right), 0\right), & \delta_{2, \mathrm{n}}=\min \left(\frac{1}{2}-W\left(\bar{P}_{2, \mathrm{n}}\right), 0\right) .
\end{array}
$$

The scheme contains a combination of at most six flux differences, three in $x$-direction and three in $y$-direction. Consequently, the discretisation stencil involves the twelve fluxes and nine grid points indicated in Fig. 4. 
It is instructive to consider the constant coefficient case, i.e., we assume that $u, v$ and $\varepsilon$ are constant. Using the property $W(z)+W(-z)=1$, we can show that the scheme (6.5) reduces to

$$
\begin{aligned}
\left(\frac{1}{2}+\right. & \left.W\left(\left|P_{2}\right|\right)\right) \delta_{x} F_{1, \mathrm{C}}^{\mathrm{h}}+\left(\frac{1}{2}-W\left(\left|P_{2}\right|\right)\right) \delta_{x} F_{1, \mathrm{uy}, \mathrm{C}}^{\mathrm{h}}+\left(\frac{1}{2}+W\left(\left|P_{1}\right|\right)\right) \delta_{y} F_{2, \mathrm{C}}^{\mathrm{h}} \\
& +\left(\frac{1}{2}-W\left(\left|P_{1}\right|\right)\right) \delta_{y} F_{2, \mathrm{ux}, \mathrm{C}}^{\mathrm{h}} \\
= & \left(W\left(\left|P_{1}\right|\right)+W\left(\left|P_{2}\right|\right)\right) s_{\mathrm{C}}+\left(\frac{1}{2}-W\left(\left|P_{2}\right|\right)\right) s_{\mathrm{uy}, \mathrm{C}}+\left(\frac{1}{2}-W\left(\left|P_{1}\right|\right)\right) s_{\mathrm{ux}, \mathrm{C}}
\end{aligned}
$$

where $\boldsymbol{x}_{\mathrm{ux}, \mathrm{C}}$ is the grid point located upwind (w.r.t. $u$ ) of $\boldsymbol{x}_{\mathrm{C}}$, i.e., $\boldsymbol{x}_{\mathrm{ux}, \mathrm{C}}=\boldsymbol{x}_{\mathrm{W}}$ if $u \geq 0$ and $\boldsymbol{x}_{\mathrm{ux}, \mathrm{C}}=\boldsymbol{x}_{\mathrm{E}}$ if $u<0$; likewise for $\boldsymbol{x}_{\mathrm{uy}, \mathrm{C}}$. Note that the scheme is a weighted average of flux differences at the central point $\boldsymbol{x}_{\mathrm{C}}$ and at the grid points $\boldsymbol{x}_{\mathrm{ux}, \mathrm{C}}$ and $\boldsymbol{x}_{\mathrm{uy}, \mathrm{C}}$ located upwind of $\boldsymbol{x}_{\mathrm{C}}$.

Finally, we consider two limiting cases. First,we take $u=v=0$, i.e., we consider the diffusion-reaction equation $-\varepsilon \nabla^{2} \varphi=s$. In this case, $P_{1}=P_{2}=0$ resulting in the standard central difference approximation, which can be written as

$$
\begin{gathered}
\delta_{x} F_{1, \mathrm{C}}^{\mathrm{h}}+\delta_{y} F_{2, \mathrm{C}}^{\mathrm{h}}=s_{\mathrm{C}}, \\
F_{1, \mathrm{e}}^{\mathrm{h}}=-\frac{\varepsilon}{\Delta x}\left(\varphi_{\mathrm{E}}-\varphi_{\mathrm{C}}\right), \quad \text { etc. }
\end{gathered}
$$

The numerical flux in this scheme is the central difference approximation of the diffusive flux $-\varepsilon \nabla \varphi$. The other limiting case is $\varepsilon=0$, corresponding to the advection-reaction equation $\boldsymbol{u} \cdot \nabla \varphi=s$. In this case scheme (6.7) reduces to the two-dimensional cell-vertex FVM $[18,19]$

$$
\begin{aligned}
& \frac{1}{2}\left(\delta_{x} F_{1, \mathrm{C}}^{\mathrm{h}}+\delta_{x} F_{1, \mathrm{uy}, \mathrm{C}}^{\mathrm{h}}\right)+\frac{1}{2}\left(\delta_{y} F_{2, \mathrm{C}}^{\mathrm{h}}+\delta_{y} F_{2, \mathrm{ux}, \mathrm{C}}^{\mathrm{h}}\right)=\frac{1}{2}\left(s_{\mathrm{uy}, \mathrm{C}}+s_{\mathrm{ux}, \mathrm{C}}\right), \\
& F_{1, \mathrm{e}}^{\mathrm{h}}=u \bar{\varphi}_{\mathrm{u}, \mathrm{e}}, \quad \text { etc. },
\end{aligned}
$$

cf. (5.7). The numerical flux is the upwind approximation of the advective flux $\boldsymbol{u} \varphi$ and the additional flux differences at $\boldsymbol{x}_{\mathrm{ux}, \mathrm{C}}$ and $\boldsymbol{x}_{\mathrm{uy}, \mathrm{C}}$ prevent that the scheme reduces to the first order upwind scheme.

From (6.7)-(6.9) we conclude that the constant coefficient scheme (6.7) is a weighted average of the central difference approximation (6.8) and the scheme (6.9), the weighting determined by the Peclet numbers.

\section{Extension to Time-dependent Conservation Laws}

Next, we extend the scheme to time-dependent conservation laws, restricting ourselves to one space dimension. The semidiscrete conservation law for $\varphi_{j}(t) \approx \varphi\left(x_{j}, t\right)$ reads

$$
\dot{\varphi}_{j}(t) \Delta x+F_{j+1 / 2}(t)-F_{j-1 / 2}(t)=s_{j}(t) \Delta x,
$$

where $\dot{\varphi}_{j}(t) \approx \partial \varphi / \partial t\left(x_{j}, t\right)$ and $s_{j}(t)=s\left(x_{j}, t\right)$; cf. (2.5). In the following we will omit the explicit dependence on the variable $t$. 
For the numerical flux $F_{j+1 / 2}$ in (7.1) we have two options. We can simply take the flux (5.2a) derived from the corresponding BVP (3.2), and henceforth referred to as the stationary complete flux (SCF) scheme. Alternatively, we can include $\partial \varphi / \partial t$ in the numerical flux, if we determine $F_{j+1 / 2}$ from the quasi-stationary BVP

$$
\begin{aligned}
& \frac{\partial}{\partial x}\left(u \varphi-\varepsilon \frac{\partial \varphi}{\partial x}\right)=s-\frac{\partial \varphi}{\partial t}, \quad x_{j}<x<x_{j+1}, \\
& \varphi\left(\boldsymbol{x}_{j}\right)=\varphi_{j}, \quad \varphi\left(\boldsymbol{x}_{j+1}\right)=\varphi_{j+1},
\end{aligned}
$$

thus including the time derivative in the source term. We can once more apply the theory in Sects. 3 and 4, to arrive at the following expression for the numerical flux

$$
F_{j+1 / 2}=\alpha_{j+1 / 2} \varphi_{j}-\beta_{j+1 / 2} \varphi_{j+1}+\Delta x\left(\gamma_{j+1 / 2}\left(s_{j}-\dot{\varphi}_{j}\right)+\delta_{j+1 / 2}\left(s_{j+1}-\dot{\varphi}_{j+1}\right)\right),
$$

referred to as the transient complete flux (TCF) scheme, where the coefficient $\alpha_{j+1 / 2}, \beta_{j+1 / 2}$ etc are defined in (5.2b); cf. (5.2a). A similar expression holds for the numerical flux $F_{j-1 / 2}$. Substituting these in the semidiscrete conservation law (7.1) we obtain the FV-TCF semidiscretisation, given by

$$
\begin{aligned}
& b_{\mathrm{W}, j} \dot{\varphi}_{j-1}+b_{\mathrm{C}, j} \dot{\varphi}_{j}+b_{\mathrm{E}, j} \dot{\varphi}_{j+1}-a_{\mathrm{W}, j} \varphi_{j-1}+a_{\mathrm{C}, j} \varphi_{j}-a_{\mathrm{E}, j} \varphi_{j+1} \\
& \quad=b_{\mathrm{W}, j} s_{j-1}+b_{\mathrm{C}, j} s_{j}+b_{\mathrm{E}, j} s_{j+1},
\end{aligned}
$$

with the coefficients $a_{\mathrm{W}, j}, b_{\mathrm{W}, j}$ etc. defined in (5.4).

The resulting semi-discretisation for the vector of unknowns $\varphi$ is either the ODE system

$$
\dot{\varphi} \Delta x+A \varphi=B s+b_{1},
$$

for the SCF scheme, or the implicit ODE system

$$
B \dot{\varphi}+A \varphi=B s+b_{2},
$$

for the TCF scheme, with the matrices $\boldsymbol{A}$ and $\boldsymbol{B}$ as defined in Sect. 5 and with $\boldsymbol{b}_{1}$ and $\boldsymbol{b}_{2}$ containing boundary data. In [32] we have shown that for dominant advection, i.e., $|P|$ large, the semidiscretisation (7.5b) has much smaller dissipation and dispersion errors than (7.5a), provided the solution is smooth. For high wave number solutions both semi-discretisations suffer from severe damping and dispersion. On the other hand, for dominant diffusion, dissipation and dispersion errors for both (7.5a) and (7.5b) are comparable. For time integration we require an A-stable, one-step method. Our choice is the trapezoidal rule; see, e.g., [15].

Analogous to the stationary scheme (5.3), the semidiscretisation (7.4) reduces to the central difference discretisation

$$
\dot{\varphi}_{j} \Delta x-\frac{1}{\Delta x}\left(\bar{\varepsilon}_{j+1 / 2}\left(\varphi_{j+1}-\varphi_{j}\right)-\bar{\varepsilon}_{j-1 / 2}\left(\varphi_{j}-\varphi_{j-1}\right)\right)=s_{j} \Delta x,
$$

for the diffusion-reaction equation $\varphi_{t}-\left(\varepsilon \varphi_{x}\right)_{x}=s$ and to the cell-vertex FVM

$$
\begin{array}{ll}
\frac{1}{2}\left(\dot{\varphi}_{j-1}+\dot{\varphi}_{j}\right) \Delta x+u_{j} \varphi_{j}-u_{j-1} \varphi_{j-1}=\frac{1}{2}\left(s_{j-1}+s_{j}\right) \Delta x & \text { if } u>0, \\
\frac{1}{2}\left(\dot{\varphi}_{j}+\dot{\varphi}_{j+1}\right) \Delta x+u_{j+1} \varphi_{j+1}-u_{j} \varphi_{j}=\frac{1}{2}\left(s_{j}+s_{j+1}\right) \Delta x & \text { if } u<0,
\end{array}
$$

for the advection-reaction equation $\varphi_{t}+(u \varphi)_{x}=s$. All semidiscretisations are second order accurate in space. 


\section{Numerical Examples}

In this section we apply several flux approximations to five model problems to assess their (order of) accuracy. We consider both diffusion-dominated and advection-dominated flow.

Example 1 Advection-diffusion-reaction equation with boundary layer at outflow.

We solve the BVP [34]

$$
\begin{aligned}
& \left(u \varphi-\varepsilon \varphi^{\prime}\right)^{\prime}=s, \quad 0<x<1, \\
& \varphi(0)=0, \quad \varphi(1)=1,
\end{aligned}
$$

with velocity $u(x)=1-b \sin \pi x$ and source term $s$ chosen such that the exact solution is given by

$$
\varphi(x)=a \sin (\pi x)+\frac{\mathrm{e}^{(x-1) / \varepsilon}-\mathrm{e}^{-1 / \varepsilon}}{1-\mathrm{e}^{-1 / \varepsilon}} .
$$

Note that for $0<\varepsilon \ll 1$ the solution has a thin boundary layer of width $\varepsilon$ near $x=1$. We take the following parameter values: $a=0.2, b=-0.95$ and $\varepsilon=1$ (dominant diffusion) or $\varepsilon=10^{-5}$ (dominant advection). Let $h=\Delta x=1 /(N-1)$ be the grid size, with $N$ the number of grid points. To determine the accuracy of a numerical solution we compute the average error $e_{h}:=\left\|\varphi-\varphi^{*}\right\|_{1} / N$, where $\varphi^{*}$ denotes the exact solution restricted to the grid, as a function of the reciprocal grid size $h^{-1}$. Table 1 shows $e_{h}$ and the reduction factors $e_{h} / e_{h / 2}$ for $\varepsilon=1$. Clearly, $e_{h} / e_{h / 2} \rightarrow 4$ for $h \rightarrow 0$ for both the HF and CF scheme, and consequently, both schemes display second order convergence behaviour for $h \rightarrow 0$. The numerical errors are approximately the same for both schemes. However, the situation is quite different for the case $\varepsilon=10^{-5}$ shown in Table 2 . In this case $e_{h} / e_{h / 2} \rightarrow 2$ for $h \rightarrow 0$ for the HF scheme, which means that the method is only first order convergent, in agreement with the observation that the HF-scheme reduces to the first order upwind scheme for the advection-reaction equation; see Sect. 5. The CF-scheme still displays second order convergence behaviour, which is consistent with the reduction of the CF-scheme to the scheme (5.7) for the advection-reaction equation. Obviously, the CF-solution is in this case much more accurate than the HF-solution.

Table 1 Example 1, errors for diffusion-dominated flow. Parameter values are: $a=0.2$, $b=-0.95$ and $\varepsilon=1$

\begin{tabular}{rlllll}
\hline$h^{-1}$ & \multicolumn{1}{l}{$\mathrm{CF}$} & & $\mathrm{HF}$ & \\
\cline { 2 - 3 } \cline { 5 - 6 } & $e_{h}$ & $e_{h} / e_{h / 2}$ & & $e_{h}$ & $e_{h} / e_{h / 2}$ \\
\hline 10 & $2.201 \times 10^{-3}$ & 3.69 & & $1.823 \times 10^{-3}$ & 3.81 \\
20 & $5.967 \times 10^{-4}$ & 3.84 & & $4.779 \times 10^{-4}$ & 3.90 \\
40 & $1.553 \times 10^{-4}$ & 3.92 & & $1.224 \times 10^{-4}$ & 3.95 \\
80 & $3.963 \times 10^{-5}$ & 3.96 & & $3.098 \times 10^{-5}$ & 3.97 \\
160 & $1.001 \times 10^{-5}$ & 3.98 & & $7.794 \times 10^{-6}$ & 3.99 \\
320 & $2.515 \times 10^{-6}$ & 3.99 & & $1.955 \times 10^{-6}$ & 3.99 \\
640 & $6.303 \times 10^{-7}$ & 3.99 & & $4.894 \times 10^{-7}$ & 4.00 \\
1280 & $1.578 \times 10^{-7}$ & & & $1.224 \times 10^{-7}$ & \\
\hline
\end{tabular}


Table 2 Example 1, errors for advection-dominated flow. Parameter values are: $a=0.2$, $b=-0.95$ and $\varepsilon=10^{-5}$

\begin{tabular}{rlllll}
\hline$h^{-1}$ & \multicolumn{1}{l}{ CF } & & HF & \\
\cline { 2 - 3 } \cline { 5 - 6 } & $e_{h}$ & $e_{h} / e_{h / 2}$ & & $e_{h}$ & $e_{h} / e_{h / 2}$ \\
\hline 10 & $2.146 \times 10^{-3}$ & 3.82 & & $1.977 \times 10^{-2}$ & 1.86 \\
20 & $5.613 \times 10^{-4}$ & 3.91 & & $1.061 \times 10^{-2}$ & 1.93 \\
40 & $1.436 \times 10^{-4}$ & 3.95 & & $5.504 \times 10^{-3}$ & 1.97 \\
80 & $3.632 \times 10^{-5}$ & 3.98 & & $2.801 \times 10^{-3}$ & 1.99 \\
160 & $9.121 \times 10^{-6}$ & 4.00 & & $1.411 \times 10^{-3}$ & 2.00 \\
320 & $2.280 \times 10^{-6}$ & 4.02 & & $7.070 \times 10^{-4}$ & 2.01 \\
640 & $5.669 \times 10^{-7}$ & 4.05 & & $3.525 \times 10^{-4}$ & 2.02 \\
1280 & $1.399 \times 10^{-7}$ & & $1.746 \times 10^{-4}$ & \\
\hline
\end{tabular}

Example 2 Advection-diffusion-reaction equation with interior layer.

We solve the BVP [17]

$$
\begin{gathered}
\left(u \varphi-\varepsilon \varphi^{\prime}\right)^{\prime}=s, \quad 0<x<1, \\
\varphi(0)=\varphi^{\prime}(1)=0,
\end{gathered}
$$

where the velocity $u$ and the source term $s$ are given by

$$
u(x)=(1+x)^{3}, \quad s(x)=\frac{s_{\max }}{1+s_{\max }(2 x-1)^{2}},
$$

respectively. The velocity is a smoothly varying function of $x$ whereas the source term has a sharp peak at $x=\frac{1}{2}$, causing a steep interior layer, provided $0<\varepsilon \ll 1$; see Fig. 5 .

For this BVP there is no exact solution available. In order to assess the order of accuracy of both schemes, we compute numerical approximations of $\varphi\left(\frac{1}{2}\right)$ with increasingly smaller grid sizes and apply Richardson extrapolation to these results; see e.g. [22]. More precisely, let

$$
\varphi\left(\frac{1}{2}\right)=\varphi_{h}+e_{h}=\varphi_{h / 2}+e_{h / 2}=\varphi_{h / 4}+e_{h / 4}, \quad h=\Delta x
$$

where $\varphi_{h}$ denotes the numerical approximation of $\varphi\left(\frac{1}{2}\right)$ computed with grid size $h$ and $e_{h}$ the corresponding (global) discretisation error, etc. Assuming the following error expansion

$$
e_{h}=C h^{p}+\mathcal{O}\left(h^{q}\right), \quad q>p,
$$

we can derive the following relation for the order of accuracy $p$ :

$$
2^{p} \doteq \frac{\varphi_{h / 2}-\varphi_{h}}{\varphi_{h / 4}-\varphi_{h / 2}}=: r_{h}
$$

The $r_{h}$-values are presented in Table 3. From this table it is evident that for dominant diffusion, i.e., $\varepsilon=10^{-1}$, both the HF and CF scheme are second order convergent for $h \rightarrow 0$. On the other hand, for dominant advection, i.e., $\varepsilon=10^{-8}$, the HF scheme shows first order convergence for $h \rightarrow 0$, whereas the CF scheme is still second order convergent. The large entries for $h^{-1}=10,20$ in the last column of the table indicate that the approximation (8.7) is not yet valid, or equivalently, the higher order terms in (8.6) can not be neglected, and 

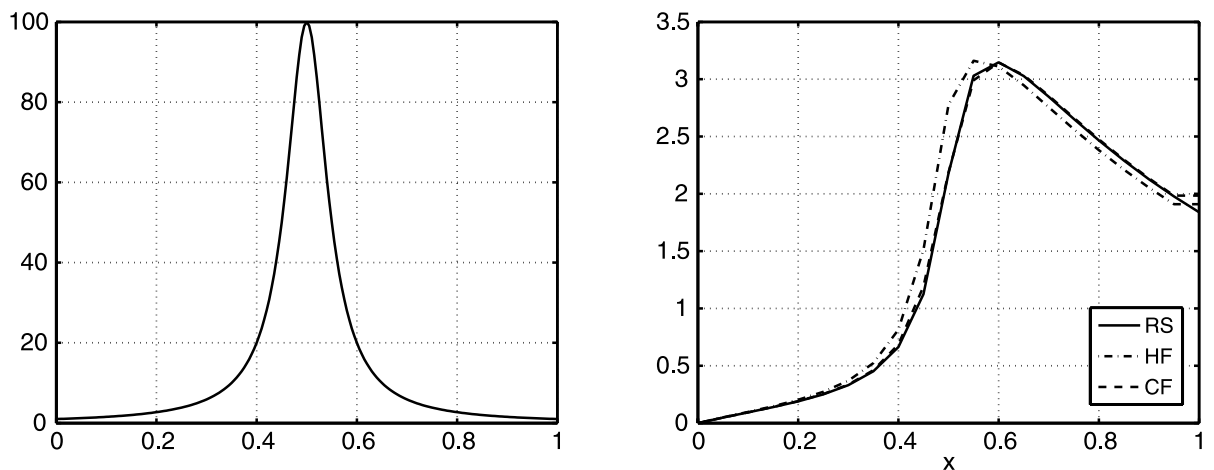

Fig. 5 Example 2, the source term $s$ (left) and the corresponding (numerical) solution (right) of (8.3). Parameter values are $\varepsilon=10^{-8}, s_{\max }=10^{2}$ and $h^{-1}=20$

Table 3 Example 2, the $r_{h}$-values as a function of $h^{-1}$, for maximum source term $s_{\max }=10^{2}$

\begin{tabular}{|c|c|c|c|c|}
\hline \multirow[t]{2}{*}{$h^{-1}$} & \multicolumn{2}{|c|}{$\varepsilon=10^{-1}$} & \multicolumn{2}{|c|}{$\varepsilon=10^{-8}$} \\
\hline & $\overline{\mathrm{HF}}$ & $\mathrm{CF}$ & $\overline{\mathrm{HF}}$ & $\mathrm{CF}$ \\
\hline 10 & 4.41 & 6.76 & 2.39 & $2.36 \times 10^{1}$ \\
\hline 20 & 4.54 & 6.00 & 1.97 & $-2.92 \times 10^{2}$ \\
\hline 40 & 4.08 & 3.65 & 1.96 & 2.57 \\
\hline 80 & 4.02 & 3.62 & 1.98 & 4.00 \\
\hline 160 & 4.00 & 3.77 & 1.99 & 4.00 \\
\hline 320 & 4.00 & 3.88 & 1.99 & 4.00 \\
\hline 640 & 4.00 & 3.94 & 2.00 & 4.00 \\
\hline 1280 & 4.00 & 3.97 & 2.00 & 4.00 \\
\hline
\end{tabular}

more importantly, that the CF-solution is already rather accurate even on these coarse grids. This is confirmed in Fig. 5 which shows the HF and CF solutions compared to the reduced solution (RS) of the problem $(u \varphi)^{\prime}=s, \varphi(0)=0$ on a rather coarse grid $\left(h^{-1}=20\right)$.

Example 3 Advection-diffusion equation with steep inlet profile and rotating flow.

Consider the following boundary value problem $[17,26]$

$$
\begin{array}{cl}
\nabla \cdot(\boldsymbol{u} \varphi-\varepsilon \nabla \varphi)=0, & -1<x<1,0<y<1, \\
\varphi(x, 0)=1+\tanh (\alpha(2 x+1)), & -1 \leq x \leq 0 \text { (inlet), } \\
\frac{\partial \varphi}{\partial y}(x, 0)=0, & 0<x \leq 1 \text { (outlet), } \\
\varphi(x, y)=1-\tanh (\alpha), & (x= \pm 1,0 \leq y \leq 1) \text { and }(-1 \leq x \leq 1, y=1),
\end{array}
$$

where the (solenoidal) velocity field is given by

$$
\boldsymbol{u}:=(u, v)=\left(2 y\left(1-x^{2}\right),-2 x\left(1-y^{2}\right)\right) .
$$

A steep interior layer is specified at the inlet, which should be advected with the rotating flow, at least for $\varepsilon$ sufficiently small. For $\varepsilon=0$ the outlet profile should be the exact mirror image of the interior layer at the inlet, whereas for small $\varepsilon>0$ the outlet profile is less steep 

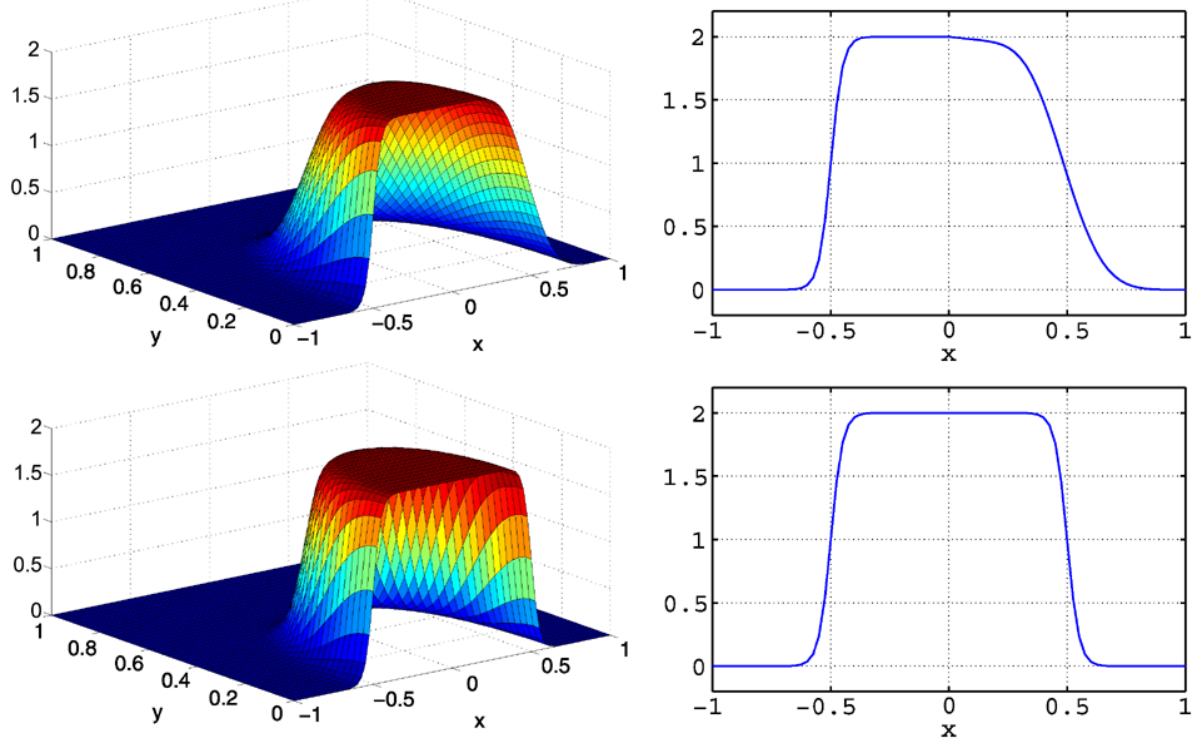

Fig. 6 Example 3, solution of boundary value problem (8.8) for $\alpha=10$ and $\varepsilon=10^{-8}$ computed with $\Delta x=\Delta y=2.5 \times 10^{-2}$. Top: HF scheme, bottom: CF scheme

Table 4 Example 3, the $r_{h}$-values as a function of $h^{-1}$ for $\alpha=10$

\begin{tabular}{rlrlll}
\hline$h^{-1}$ & \multicolumn{2}{l}{$\varepsilon=10^{-2}$} & & & \multicolumn{2}{l}{$\varepsilon=10^{-8}$} & \\
\cline { 2 - 3 } & HF & CF & & HF & CF \\
\hline 20 & 3.12 & -1.93 & & 1.49 & 5.73 \\
40 & 3.72 & 1.97 & 2.26 & 4.42 \\
80 & 3.93 & 3.07 & 3.15 & 4.11 \\
160 & 3.98 & 3.56 & & 3.29 & 4.04 \\
320 & 4.00 & 3.78 & & 2.77 & 4.01 \\
640 & 4.00 & 3.89 & & 2.38 & 4.01 \\
\hline
\end{tabular}

due to diffusion. Numerical solutions of (8.8) for $\varepsilon=10^{-8}$ and $\alpha=10$ are displayed in Fig. 6. Clearly, the HF solution is far too smooth, due to numerical diffusion, whereas the outlet profile of the CF solution is hardly distorted. Apparently, inclusion of the cross flux terms in the numerical fluxes reduces numerical diffusion considerably.

To determine the order of accuracy, we apply Richardson extrapolation to numerical approximations of $\varphi\left(\frac{1}{2}, \frac{1}{2}\right)$, i.e., we compute the quotients $r_{h}$ defined in (8.7) with $\varphi_{h}$ the numerical approximation of $\varphi\left(\frac{1}{2}, \frac{1}{2}\right)$ computed with grid sizes $\Delta x=\Delta y=h$. The results are presented in Table 4. From this we may conclude, that the CF scheme is second order convergent for $h \rightarrow 0$ for both diffusion dominated flow $\left(\varepsilon=10^{-2}\right)$ and advection dominated flow $\left(\varepsilon=10^{-8}\right)$. The HF scheme however is second order for diffusion dominated flow only; it reduces to first order for dominant advection. 

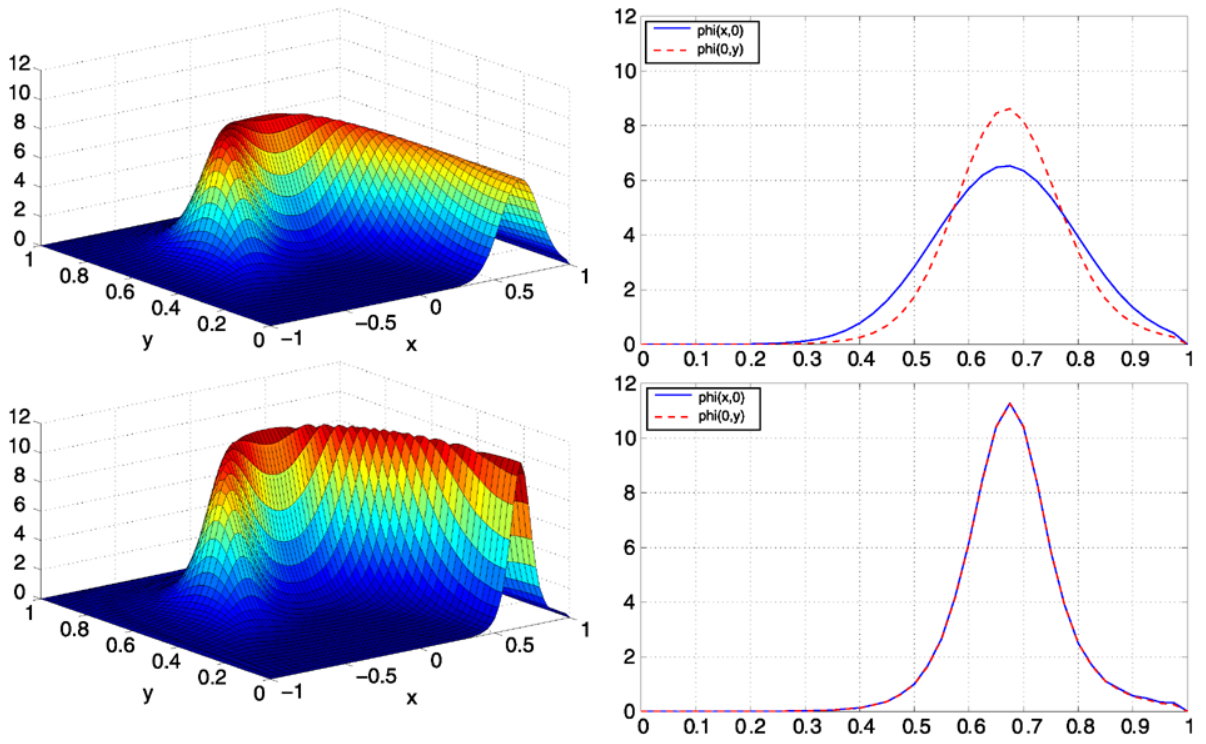

Fig. 7 Example 4, solution of boundary value problem (8.10) for $\beta=10, s_{\max }=10^{2}$ and $\varepsilon=10^{-8}$ computed with $\Delta x=\Delta y=2.5 \times 10^{-2}$. Top: HF scheme, bottom: CF scheme

Example 4 Advection-diffusion-reaction equation with rotating flow.

Consider the following modification of the BVP (8.8) in Example 3, i.e.,

$$
\begin{array}{cl}
\nabla \cdot(\boldsymbol{u} \varphi-\varepsilon \nabla \varphi)=s, & -1<x<1,0<y<1, \\
\varphi(x, 0)=0, & -1 \leq x \leq 0 \text { (inlet), } \\
\frac{\partial \varphi}{\partial y}(x, 0)=0, & 0<x \leq 1 \text { (outlet), } \\
\varphi(x, y)=0, & (x= \pm 1,0 \leq y \leq 1) \text { and }(-1 \leq x \leq 1, y=1),
\end{array}
$$

where the velocity field is defined in (8.9) and where the source term is given by

$$
\begin{aligned}
& s(x, y)=\frac{1}{2} \frac{s_{\max }}{1+s_{\max }\left(x^{\prime}\right)^{2}}\left(1-\tanh ^{2}\left(\beta\left(\frac{1}{2} \sqrt{2}-y^{\prime}\right)\right)\right), \\
& \left(x^{\prime}, y^{\prime}\right):=\frac{1}{2} \sqrt{2}(x+y,-x+y) .
\end{aligned}
$$

In this example there is no inlet profile, instead, the solution is generated by a source term which has a sharp peak near $\left(x^{\prime}, y^{\prime}\right)=\left(0, \frac{1}{2} \sqrt{2}\right)$, i.e., at $(x, y)=\left(-\frac{1}{2}, \frac{1}{2}\right)$. Thus, the solution is created in the second quadrant $(x<0, y>0)$. Numerical solutions for $\beta=10, s_{\max }=$ $10^{2}$ and $\varepsilon=10^{-8}$ are given in Fig. 7. The solution profile $\varphi(0, y)$ along the centre line is compared with the solution $\varphi(x, 0)(0 \leq x \leq 1)$ at the outlet. Since $\varepsilon=10^{-8}$ and virtually $s=0$ in the first quadrant $(x, y>0)$, advection is the only relevant transport term in (8.10a), implying that both solution profiles should be almost the same. This is clearly true for the CF solution, however, the HF outlet profile is too much smeared out, this due to cross wind diffusion. 
Table 5 Example 5, errors for the advection-reaction equation. Parameter values are: $u=0.95$, and $\tau=4 \times 10^{-2}$

\begin{tabular}{rlllll}
\hline$h^{-1}$ & TCF & & SCF & \\
\cline { 2 - 3 } \cline { 5 - 6 } & $e_{h}$ & $e_{h} / e_{h / 2}$ & & $e_{h}$ & $e_{h} / e_{h / 2}$ \\
\hline 20 & $4.645 \times 10^{-2}$ & 1.64 & & $5.743 \times 10^{-2}$ & 1.19 \\
40 & $2.831 \times 10^{-2}$ & 1.97 & & $4.837 \times 10^{-2}$ & 1.21 \\
80 & $1.436 \times 10^{-2}$ & 2.75 & & $4.011 \times 10^{-2}$ & 1.30 \\
160 & $5.221 \times 10^{-3}$ & 3.48 & & $3.078 \times 10^{-2}$ & 1.40 \\
320 & $1.502 \times 10^{-3}$ & 3.83 & & $2.198 \times 10^{-2}$ & 1.52 \\
640 & $3.918 \times 10^{-4}$ & 3.95 & & $1.445 \times 10^{-2}$ & 1.65 \\
1280 & $9.923 \times 10^{-5}$ & & & $8.742 \times 10^{-3}$ & \\
\hline
\end{tabular}

Example 5 Advection-reaction equation.

Consider the following model IBVP for hyperbolic-relaxation equations [29]

$$
\begin{aligned}
& \frac{\partial \varphi}{\partial t}+\frac{\partial}{\partial x}(u \varphi)=-\frac{1}{\tau} \varphi(1-\varphi), \quad 0<x<1, t>0, \\
& \varphi(x, 0)=a(x)=0.8, \quad 0<x<1, \\
& \varphi(0, t)=b(t)=0.8+0.2 \sin (2 \pi t), \quad t>0,
\end{aligned}
$$

where $u>0$ is the flow velocity and where $\tau \ll 1$ is a relaxation time. We choose $u \tau \ll 1$, which means that the time scale of advection is much larger than $\tau$. Using the method of characteristics, see e.g. [12], we can solve the IBVP (8.12) to find

$$
\varphi(x, t)= \begin{cases}\left(1+\left(\frac{1}{\varphi_{1}}-1\right) \mathrm{e}^{t / \tau}\right)^{-1}, & \varphi_{1}=a(x-u t) \text { for } x \geq u t, \\ \left(1+\left(\frac{1}{\varphi_{2}}-1\right) \mathrm{e}^{x /(u \tau)}\right)^{-1}, & \varphi_{2}=b(t-x / u) \text { for } x<u t .\end{cases}
$$

The oscillating boundary condition $(8.12 \mathrm{c})$ generates a wave propagating in the positive $x$-direction. The ODE $\mathrm{d} \varphi / \mathrm{d} t=-\frac{1}{\tau} \varphi(1-\varphi)$ corresponding to (8.12a), which holds along the characteristics, has a stable equilibrium $\varphi=0$ and an unstable $\varphi=1$. The effect of the source term is therefore that the constant state ahead of the wave approaches 0 , whereas a narrow peak near $\varphi(x, t)=1$ is created.

We have computed numerical solutions of (8.12) using the SCF and TCF scheme, in combination with the trapezoidal rule for time integration. We choose the following parameter values: $u=0.95$ and $\tau=4 \times 10^{-2}$, and moreover, we take $\Delta x=\Delta t=: h$. To determine the accuracy of a numerical solution we compute the average error $e_{h}:=h\left\|\varphi-\varphi^{*}\right\|_{1}$ at $t=0.5$, where $\varphi^{*}$ denotes the exact solution restricted to the grid, as a function of the reciprocal grid size $h^{-1}$. Table 5 shows $e_{h}$ and the reduction factors $e_{h} / e_{h / 2}$. Clearly, for the TCF scheme $e_{h} / e_{h / 2} \rightarrow 4$ for $h \rightarrow 0$, implying that the discretisation method is second order. However, the SCF discretisation does not even display first order convergence, and the corresponding solutions have a much larger discretisation error due to dissipation errors; see [32].

As an example, we show in Fig. 8 the numerical solutions computed with the TCF and SCF scheme, respectively, computed for $h^{-1}=1280$. Obviously, the SCF scheme suffers from severe damping, resulting in a far too small maximum, whereas for the TCF scheme the peak near the maximum is well resolved. 

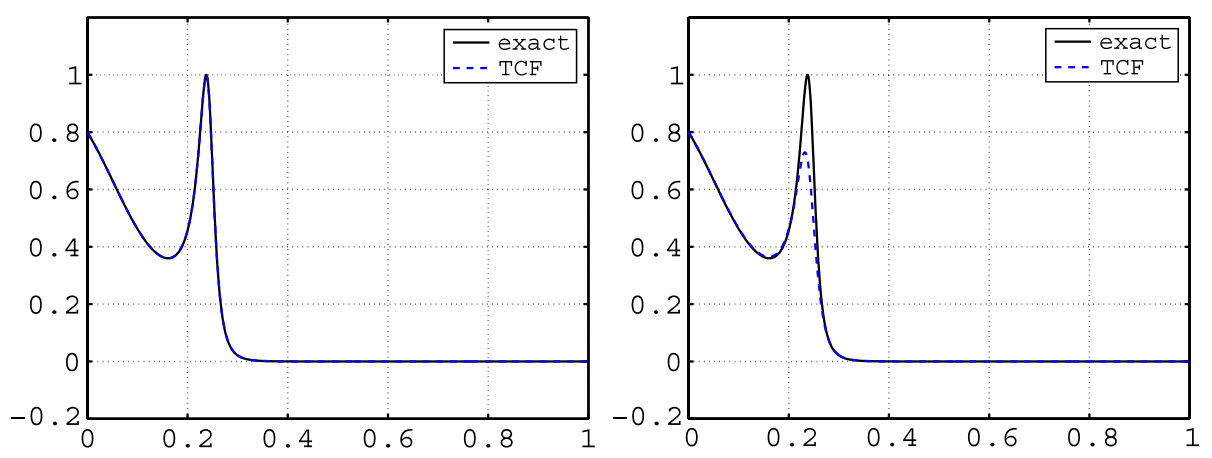

Fig. 8 Example 5, numerical solution of IBVP (8.12) at $t=0.5$ for $u=0.95$ and $\tau=4 \times 10^{-2}$ computed with $h^{-1}=1280$. Left: TSC scheme, right: SCF scheme

\section{Summary, Conclusions and Future Research}

We have derived an integral representation for the flux of the one-dimensional advectiondiffusion-reaction equation from a local BVP for the entire equation, including the source term. As a consequence, the flux consists of two parts, i.e., a homogeneous and an inhomogeneous part, corresponding to the homogeneous and particular solution of the BVP, respectively. A new representation of the inhomogeneous flux in terms of a Green's function is given. Combining this integral representation with suitable quadrature rules, we could derive expressions for the numerical flux. Obviously, also the numerical flux consists of a homogeneous and an inhomogeneous part. The inhomogeneous part turns out to be very important for dominant advection, since it ensures that the flux approximation remains second order. The resulting finite volume scheme turns out to be second order accurate, uniformly in the local Peclet numbers, virtually never generates spurious oscillations, and moreover, has only a three-point coupling.

For two-dimensional problems we have to include the cross flux term in the inhomogeneous flux. This means that, say for the discretisation of the $x$-component $f_{1}$ of the flux, we have to solve a quasi-one dimensional BVP, where the source term contains the cross flux term $\partial f_{2} / \partial y$; see (6.1). The finite volume method obtained this way usually gives very accurate approximations of steep (interior) layers, in case of dominant advection, and has a compact nine-point stencil. However, (small) spurious oscillations cannot always be excluded. A remedy to this could be to take a combination of the complete and the homogeneous fluxes. This is topic of further research.

As a second extension, we applied the complete flux scheme to time-dependent problems. The key idea is to include the time derivative in the inhomogeneous flux, i.e., the flux is determined from a quasi-stationary BVP where the source term contains the time derivative. The resulting semidiscretisation is an implicit ODE system and usually has small dissipation and dispersion errors, see [32]. Spurious oscillations in the semidiscrete solution can occur, and have to be controlled by a (dissipative) time integration method.

Currently, we are extending our research in the following directions. First, we combine the integral representation of the flux with Gauss quadrature rules to derive higher order schemes; first results are presented in [2]. Second, we analyse several time integration methods when combined with the complete flux scheme; in particular we investigate exponential time integrators. Finally, we extend the complete flux scheme to advection-diffusionreaction systems, where the equations are coupled through an advection and a diffusion matrix. Preliminary results are promising and will be reported elsewhere. 
Open Access This article is distributed under the terms of the Creative Commons Attribution Noncommercial License which permits any noncommercial use, distribution, and reproduction in any medium, provided the original author(s) and source are credited.

\section{References}

1. Allen, D., Southwell, R.: Relaxation methods applied to determining the motion, in two dimensions, of a viscous fluid past a fixed cylinder. Quart. J. Mech. Appl. Math. 8, 129-145 (1955)

2. Anthonissen, M.J.H., Ten Thije Boonkkamp, J.H.M.: A compact high order finite volume scheme for advection-diffusion-reaction equations. In: Numerical Analysis and Applied Mathematics: International Conference on Numerical Analysis and Applied Mathematics 2009. AIP Conference Proceedings, vol. 1168, pp. 410-414. American Institute of Physics, New York (2009)

3. Bank, R.E., Rose, D.J., Fichtner, W.: Numerical methods for semiconductor device simulation. IEEE Trans. Electron Devices 30, 1031-1041 (1983)

4. Brezzi, F., Marini, L.D., Pietra, P.: Two-dimensional exponential fitting and applications to drift-diffusion models. SIAM J. Numer. Anal. 26, 1342-1355 (1989)

5. Doolan, E.P., Miller, J.J.H., Schilders, W.H.A.: Uniform Numerical Methods for Problems with Initial and Boundary Layers. Boole Press, Dublin (1980)

6. El-Mistikawy, T.M., Werle, M.J.: Numerical methods for boundary layers with blowing- the exponential box scheme. AIAA J. 16, 749-751 (1978)

7. Eymard, R., Gallouët, T., Herbin, R.: Finite volume methods. In: Ciarlet, P.G., Lions, J.L. (eds.) Handbook of Numerical Analysis. vol. VII, pp. 713-1020. North-Holland, Amsterdam (2000)

8. Van't Hof, B., Ten Thije Boonkkamp, J.H.M., Mattheij, R.M.M.: Discretisation of the stationary convection-diffusion-reaction equation. Numer. Methods Partial Differ. Equ. 14, 607-625 (1998)

9. Hundsdorfer, W., Verwer, J.G.: Numerical Solution of Time-dependent Advection-Diffusion-Reaction Equations. Springer, Berlin (2003)

10. Il'in, A.M.: Differencing scheme for a differential equation with a small parameter affecting the highest derivative. Mat. Zametki 6, 237-248 (1969) (in Russian)

11. Jerome, J.W.: Analysis of Charge Transport. Springer, Berlin (1996)

12. Kevorkian, J.: Partial Differential Equations, Analytical Solution Techniques. Wadsworth \& Brooks, Pacific Grove (1990)

13. LeVeque, R.J.: Finite Volume Methods for Hyperbolic Problems, Cambridge Texts in Applied Mathematics. Cambridge University Press, Cambridge (2002)

14. Markowich, P.A., Ringerhofer, C.A., Schmeiser, C.: Semiconductor Equations. Springer, Vienna (1990)

15. Mattheij, R.M.M., Rienstra, S.W., Ten Thije Boonkkamp, J.H.M.: Partial Differential Equations, Modeling, Analysis, Computation. SIAM, Philadelphia (2005)

16. Miller, J.J.H.: On the inclusion of the recombination term in discretizations of the semicoductor device equations. Math. Comput. Simul. 28, 1-11 (1986)

17. Morton, K.W.: Numerical Solution of Convection-Diffusion Problems. Applied Mathematics and Mathematical Computation, vol. 12. Chapman \& Hall, London (1996)

18. Morton, K.W., Süli, E.: Finite volume methods and their analysis. IMA J. Numer. Anal. 11, 241-260 (1991)

19. Morton, K.W., Stynes, M., Süli, E.: Analysis of a cell-vertex finite volume method for convectiondiffusion problems. Math. Comput. 66, 1389-1406 (1997)

20. Patankar, S.V.: Numerical Heat Transfer and Fluid Flow, Series in Computational Methods in Mechanics and Thermal Sciences. Hemishere Publishing Corporation, New York (1980)

21. Poinsot, T., Veynante, D.: Theoretical and Numerical Combustion, 2nd edn. Edwards, Philadelphia (2005)

22. Quarteroni, A., Sacco, R., Saleri, F.: Numerical Mathematics. Texts in Applied Mathematics, vol. 37. Springer, New York (2000)

23. Raizer, Yu.P.: Gas Discharge Physics. Springer, Berlin (1991)

24. Schilders, W.H.A.: Application of exponential fitting techniques to semiconductor device simulation. In: Miller, J.H.H. (ed.) Computational Methods for Boundary and Interior Layers, pp. 175-202. Boole Press, Dublin (1991)

25. Shu, C.-W.: Essentially non-oscillatory and weighted essentially non-oscillatory schemes for hyperbolic conservation laws. In: Quarteroni, A. (ed.) Advanced Numerical Approximation of Nonlinear Hyperbolic Equations. Lecture Notes in Mathematics, vol. 1697, pp. 325-432. Springer, Berlin (1998)

26. Smith, R.M., Hutton, A.G.: The numerical treatment of advection: a performance comparison of current methods. Numer. Heat Transf. 5, 439-461 (1982) 
27. Spalding, D.B.: A novel finite difference formulation for differential expressions involving both first and second derivatives. Int. J. Numer. Methods Eng. 4, 551-559 (1972)

28. Spanier, J., Oldham, K.B.: An Atlas of Functions. Springer, Berlin (1987)

29. Suzuki, Y., Khieu, L., Van Leer, B.: CFD by first order PDEs. Contin. Mech. Thermodyn. 21, 445-465 (2009)

30. Thiart, G.D.: Finite difference scheme for the numerical solution of fluid flow and heat transfer problems on nonstaggered grids. Numer. Heat Transf., Part B 17, 41-62 (1990)

31. Thiart, G.D.: Improved finite-difference scheme for the solution of convection-diffusion problems with the SIMPLEN algorithm. Numer. Heat Transf., Part B 18, 81-95 (1990)

32. Ten Thije Boonkkamp, J.H.M., Anthonissen, M.J.H.: Extension of the complete flux scheme to timedependent conservation laws. In: Proceedings ENUMATH 2009 (2010, to appear)

33. Ten Thije Boonkkamp, J.H.M., Schilders, W.H.A.: An exponential fitting scheme for the electrothermal device equations specifically for the simulation of avalanche generation. COMPEL 12, 95-111 (1993)

34. Wesseling, P.: Principles of Computational Fluid Dynamics. Springer Series in Computational Mathematics, vol. 29. Springer, Berlin (2000) 